ARTICLE

https://doi.org/10.1038/s41467-019-10694-z

OPEN

\title{
Mechanisms of $\mathrm{Ca}^{2+} /$ calmodulin-dependent kinase II activation in single dendritic spines
}

Jui-Yun Chang ${ }^{1,2}$, Yoshihisa Nakahata ${ }^{2}$, Yuki Hayano (D) ${ }^{2}$ \& Ryohei Yasuda (D) ${ }^{2}$

CaMKIl $\alpha$ plays an essential role in decoding $\mathrm{Ca}^{2+}$ signaling in spines by acting as a leaky $\mathrm{Ca}^{2+}$ integrator with the time constant of several seconds. However, the mechanism by which CaMKIl $\alpha$ integrates $\mathrm{Ca}^{2+}$ signals remains elusive. Here, we imaged CaMKIl $\alpha-\mathrm{CaM}$ association in single dendritic spines using a new FRET sensor and two-photon fluorescence lifetime imaging. In response to a glutamate uncaging pulse, CaMKIl $\alpha-\mathrm{CaM}$ association increases in $\sim 0.1 \mathrm{~s}$ and decays over $\sim 3 \mathrm{~s}$. During repetitive glutamate uncaging, which induces spine structural plasticity, CaMKIl $\alpha-\mathrm{CaM}$ association did not show further increase but sustained at a constant level. Since CaMKIl $\alpha$ activity integrates $\mathrm{Ca}^{2+}$ signals over $\sim 10 \mathrm{~s}$ under this condition, the integration of $\mathrm{Ca}^{2+}$ signal by CaMKll $\alpha$ during spine structural plasticity is largely due to $\mathrm{Ca}^{2+} / \mathrm{CaM}$-independent, autonomous activity. Based on these results, we propose a simple kinetic model of CaMKIl $\alpha$ activation in dendritic spines. 
C alcium $\left(\mathrm{Ca}^{2+}\right) /$ calmodulin-dependent kinase II (CaMKII), a serine/threonine kinase, is critical for various forms of synaptic plasticity that underlie learning and memory. CaMKII is composed of 12 subunits, each of which is a kinase that is activated by the binding of $\mathrm{Ca}^{2+} /$ calmodulin $(\mathrm{CaM})^{1}$. The most abundant subunit in the forebrain, CaMKIIa, is required for LTP, spine structural LTP (sLTP) and spatial learning ${ }^{2-5}$. In addition to $\mathrm{Ca}^{2+} / \mathrm{CaM}$ binding, CaMKIIa activity is regulated by autophosphorylation at multiple sites. Autophosphorylation at Thr286 prolongs CaMKIIa activity ${ }^{6-8}$, permitting the integration of $\mathrm{Ca}^{2+}$ transients to facilitate the induction of spine plasticity ${ }^{9,10}$. Disruption of this phosphorylation in Camk $2 a^{\mathrm{T} 286 \mathrm{~A}}$ knock-in mice impairs LTP, sLTP, and spatial learning and memory ${ }^{10,11}$. It is known that phosphorylation at Thr286 causes an enhancement in binding affinity to $\mathrm{Ca}^{2+} / \mathrm{CaM}^{7,12}$ as well as induces a $\mathrm{Ca}^{2+} / \mathrm{CaM}$-independent, autonomous kinase activity state ${ }^{13,14}$. This autonomous activity of CaMKIIa is thought to be important for the induction and the maintenance of LTP ${ }^{14}$. CaMKIIa is additionally regulated by autophosphorylation at Thr305 and Thr306, which inhibit binding of $\mathrm{Ca}^{2+} / \mathrm{CaM}$ to CaMKIIa ${ }^{15,16}$.

CaMKIIa activity in response to $\mathrm{Ca}^{2+}$ elevations in dendritic spines can be measured by a fluorescence resonance energy transfer (FRET) sensor Camuia in combination with 2-photon fluorescence lifetime imaging (2pFLIM $)^{6,10,17}$. A brief pulse of two-photon glutamate uncaging induces a transient $\mathrm{Ca}^{2+}$ elevation, lasting $\sim 100 \mathrm{~ms}$, in the stimulated spine ${ }^{6,10,18}$. This causes a rapid CaMKIIa activation, which peaks within $\sim 0.5 \mathrm{~s}$ and then decays over $\sim 10 \mathrm{~s}$, in the stimulated spine ${ }^{10}$. In response to a repetitive glutamate uncaging $(\sim 0.5 \mathrm{~Hz})$, which induces LTP in the stimulated spine $e^{5,6}$, CaMKIIa activity increases in a stepwise manner, following each uncaging pulse until plateauing within $\sim 10 \mathrm{~s}$ (ref. ${ }^{10}$ ). After the cessation of glutamate uncaging, CaMKIIa activity decayed with time constants of $\sim 6 \mathrm{~s}$ and $\sim 1 \mathrm{~min}$ (ref. ${ }^{10}$ ). These experiments suggest that CaMKIIa is a leaky integrator of $\mathrm{Ca}^{2+}$ signals ${ }^{10}$.

Camuia measures the conformation change of CaMKIIa associated with its activation by both $\mathrm{Ca}^{2+} / \mathrm{CaM}$ binding and Thr286 autophosphorylation 6,17 . Previous studies using this sensor suggest that the optimal integration of $\mathrm{Ca}^{2+}$ signals by CaMKIIa requires Thr286 autophosphorylation, suggesting that autonomous activity may play an important role in this process $^{6,10}$. However, if an autonomous state of CaMKIIa exists in the stimulated spines, and if so, how much this state contributes to CaMKIIa activation remains elusive.

Here, we used two-photon fluorescence lifetime microscopy (2pFLIM) to probe the association between CaMKIIa and $\mathrm{Ca}^{2+} / \mathrm{CaM}$. Our results revealed that the fraction of CaMKIIa bound to $\mathrm{Ca}^{2+} / \mathrm{CaM}$ does not continue to increase with multiple $\mathrm{Ca}^{2+}$ transients during the induction of sLTP. Taken together with our previous report showing that CaMKIIa activity integrates $\mathrm{Ca}^{2+}$ signals over $\sim 10 \mathrm{~s}$ to 1 min under similar conditions ${ }^{10}$, our results suggest that the integration of $\mathrm{Ca}^{2+}$ signals depends largely on $\mathrm{Ca}^{2+} / \mathrm{CaM}$-independent, 'autonomous' activity of CaMKIIa. We propose a simple kinetic scheme of CaMKIIa activation that is consistent with our experimental results both for CaMKIIa-CaM association and for CaMKIIa activation. This model highlights that autonomous activity, but not $\mathrm{Ca}^{2+} / \mathrm{CaM}$-dependent activity, accounts for the majority of CaMKIIa activity.

\section{Results}

Sensor for association of CaMKIIa and calmodulin. To measure the association of CaMKIIa with CaM, we developed a FRET-based biosensor made of monomeric EGFP (mEGFP)CaMKIIa and mCherry-CaM (Fig. 1a) ${ }^{19}$. Biochemical cell-free assays showed that mCherry-CaM supports CaMKIIa activity
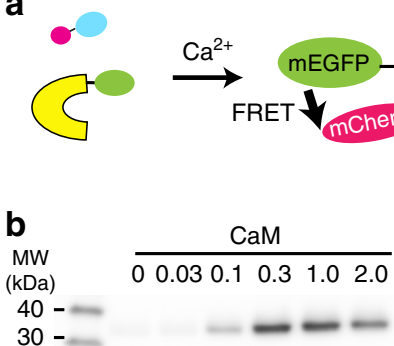

mCherry-CaM $\overline{00.03 \quad 0.10 .31 .02 .0}(\mu \mathrm{M})$ -- pSyn 1
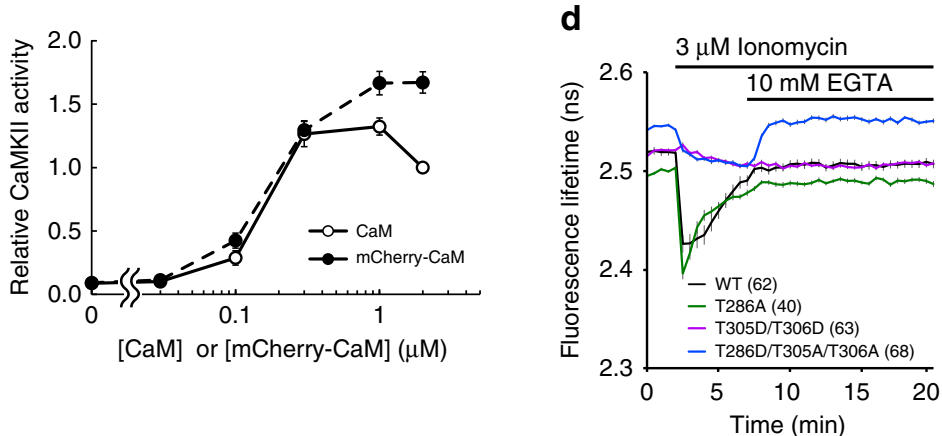
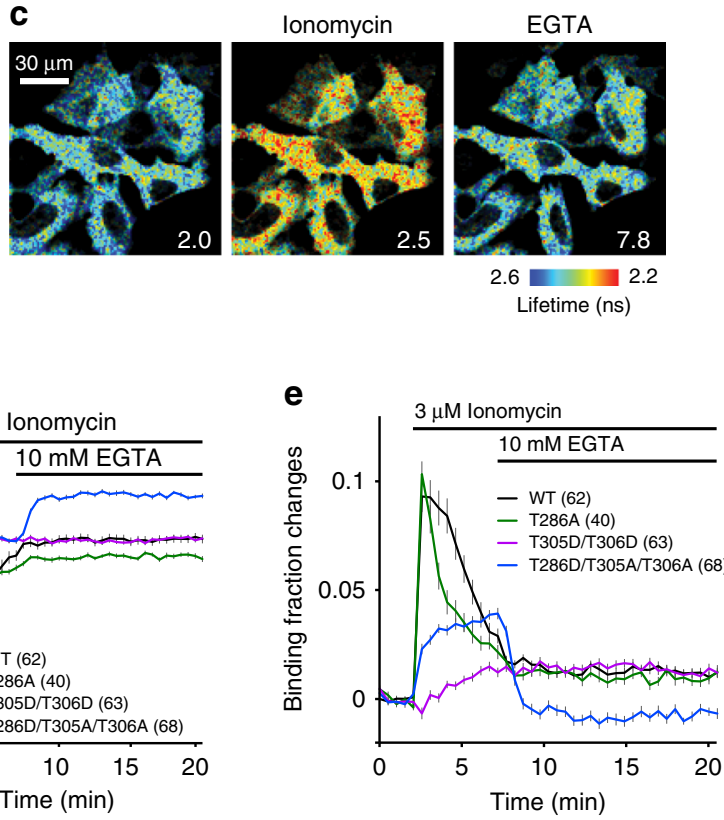

Fig. 1 Design and characterization of CaMKIl $\alpha$-CaM association sensor. a Design of a FRET sensor for CaMKIl $\alpha$-CaM association. Monomeric EGFP (mEGFP) and monomeric Cherry (mCherry) fluorescent protein are fused to the N-terminus of CaMKIl $\alpha$ and the N-terminus of CaM, respectively. b mCherry-CaM activates CaMKIl $\alpha$ to the degree similar to non-labeled CaM at different concentrations of CaM in a cell-free system. Upper panel: western blot of phosphorylated Synapsin1 peptide (pSyn1) fused to mCherry. Lower panel: quantification of pSyn1 signal from 4 experiments, normalized with the pSyn1 signal at $2 \mu \mathrm{M}$ non-labeled CaM. c Fluorescence lifetime images of CaMKIl $\alpha$-CaM association sensor expressed in HeLa cells. d Time courses of fluorescence lifetime of CaMKIl $\alpha$-CaM association sensor and its mutants (T286A, T305D/T306D and T286D/T305A/T306A) in response to bath application of ionomycin $(3 \mu \mathrm{M})$ and EGTA $(10 \mathrm{mM})$. e Time courses of changes in CaMKIl $\alpha$-CaM association calculated from d. All data are shown in mean \pm sem 
similarly to a non-labeled $\mathrm{CaM}$ at a wide range of concentrations $(0.03 \mu \mathrm{M}<[\mathrm{CaM}$ or mCherry-CaM] $<2 \mu \mathrm{M})$ (Fig. 1b), suggesting that mCherry fusion does not affect the affinity of CaM for CaMKIIa.

We further characterized the CaMKIIa-CaM association sensor in HeLa cells (Fig. 1c-e). To do so, we bath-applied an ionophore $\left(3 \mu \mathrm{M}\right.$ ionomycin) to elevate intracellular $\left[\mathrm{Ca}^{2+}\right]$, and then subsequently added EGTA to reverse the reaction. In response to the ionophore application, the CaMKIIa-CaM association sensor first showed a rapid increase in FRET signal, which decayed over a few minutes. This signal further decayed in response to extracellular EGTA application, which chelates extracellular $\left[\mathrm{Ca}^{2+}\right]$ (and thus decreasing intracellular $\left[\mathrm{Ca}^{2+}\right]$ ) (Fig. 1c-e). However, we observed a residual CaMKIIa-CaM association, which persisted more than $20 \mathrm{~min}$.

Next, we characterized the association of CaM with various CaMKIIa phosphorylation mutants. We first introduced phosphomimic mutations of inhibitory autophosphorylation sites (T305D/ T306D) to inhibit the interaction of CaM to the regulatory domain of CaMKIIa ${ }^{2,15}$. We found that the mutation largely inhibited the rapid CaM binding, consistent with the previous reports ${ }^{15}$. However, there was a small and persistent increase in FRET signal, whose amplitude and time scale are similar to that of the persistent component of the FRET signal of wildtype (WT) CaMKIIa ${ }^{\text {WT }}-\mathrm{CaM}$ association. Since the regulatory domain of T305D/T306D mutant does not have the capability to bind $\mathrm{Ca}^{2}$ $+/ \mathrm{CaM}^{2,15}$, the observed persistent CaMKIIa ${ }^{\mathrm{WT}}$-CaM association is unlikely due to the association of CaM to the regulatory domain of CaMKIIa. Phospho-dead mutation at Thr286 (T286A), the site important for autonomous CaMKII $\alpha$ activation ${ }^{8}$, accelerated the decay of FRET signal, consistent with a role phosphorylation at this site to prolong CaMKIIa activation ${ }^{10}$. A small population of CaMKIIa ${ }^{\mathrm{T} 286 \mathrm{~A}}$ mutant also exhibited persistent CaM binding, suggesting that this component is related to neither CaM binding to the regulatory domain nor T286 autophosphorylation, and thus perhaps not related to the regulation of CaMKIIa activation. Finally, we measured the binding of CaM with a phospho-mimic mutation at Thr286 (T286D). Since this mutation is known to cause inhibitory autophosphorylation at T305/T306, which inhibits $\mathrm{Ca}^{2+} / \mathrm{CaM}$ binding ${ }^{15,16}$, we introduced T305A/T306A mutation in addition to T286D (CaMKIIa $\left.{ }^{\mathrm{T} 286 \mathrm{D} / \mathrm{T} 305 \mathrm{~A} / \mathrm{T} 306 \mathrm{~A}}\right)^{20}$. In response to ionophore application, CaMKIIaT286D/T305A/T306A $\mathrm{CaM}$ association displayed a persistent increase, which was reversed by EGTA application.

Association of CaMKIIa-CaM in dendritic spines. To measure the association of CaMKIIa-CaM during the induction of spine plasticity, we biolistically transfected organotypic hippocampal slice cultures of mice with the CaMKIIa-CaM association sensor and imaged CA1 pyramidal neurons with 2pFLIM. Structural LTP (sLTP) was induced in a single spine by applying repetitive pulses $(0.49 \mathrm{~Hz}, 30$ pulses $)$ of two-photon glutamate uncaging to the spine in the absence of extracellular $\mathrm{Mg}^{2+}$ (refs. ${ }^{5,6}$ ).

We first measured CaMKIIa-CaM association during sLTP induction with a temporal resolution of $128 \mathrm{~ms} /$ frame (Fig. 2a). Binding of CaM to CaMKIIa occurs rapidly within 1 frame (128 ms) in the stimulated spine. The binding plateaued with the first glutamate uncaging pulse, and subsequent uncaging pulses did not result in a higher level of CaMKIIa-CaM association (Fig. 2b-e). The fractional change in binding of CaMKIIa to $\mathrm{Ca}^{2+} / \mathrm{CaM}$ during sLTP induction was independent of the overexpression level (Supplementary Fig. 1). After cessation of glutamate uncaging, CaM dissociated from CaMKIIa with the time constant of $3.2 \pm 0.7 \mathrm{~s}$. In addition to the fast decay, we observed a persistent component after cessation of uncaging (Fig. 2d, e). This component appeared to be not related to the binding of $\mathrm{Ca}^{2+} / \mathrm{CaM}$ to the CaM-binding domain of CaMKIIa, since a CaMKIIa mutant without binding capability (CaMKIIa ${ }^{\mathrm{T} 305 \mathrm{D} /}$ T306D) also showed this persistent component (Fig. 2f), similarly to the results in HeLa cells (Fig. 1e).

The above experiments were performed at room temperature $\left(25-27^{\circ} \mathrm{C}\right)$. At a near physiological temperature $\left(34-35^{\circ} \mathrm{C}\right)$, $\mathrm{Ca}^{2+} / \mathrm{CaM}$ dissociated faster $(\tau=0.4 \pm 0.5 \mathrm{~s}$; Supplementary Fig. 2). The temperature coefficient of the dissociation kinetics was determined to be $Q_{10}=10.3$.

To determine whether CaMKIIa-CaM association showed the integration of multiple uncaging pulses, we compared the binding induced during sLTP (trains of pulses) with the CaMKIIa-CaM association in response to a single glutamate uncaging pulse (Fig. 3). Binding of $\mathrm{Ca}^{2+} / \mathrm{CaM}$ to CaMKIIa increased rapidly in response to a single uncaging pulse, to a magnitude similar to sLTP-inducing stimulations and then decayed (Fig. 3a-c). The dissociation time constant was obtained as $\tau=2.9 \pm 0.3 \mathrm{~s}$ (Fig. 3c), a value similar to that obtained after the cessation of sLTP induction (Fig. 2e). The fraction of CaMKIIa binding to CaM was similar during trains of glutamate uncaging and in response to a single glutamate uncaging pulse (Fig. 3d). This is a sharp contrast to measurements of the active conformation CaMKIIa in spines (Fig. 3e), which shows a slower decay in response to a single uncaging pulse $(6.4 \pm 0.7 \mathrm{~s}(74 \%)$ and $92.6 \pm 50.7 \mathrm{~s}(26 \%))$, and accumulates to higher levels during trains of uncaging pulses ${ }^{10}$.

Role of Thr286 phosphorylation in CaMKIIa-CaM association. It has been reported that the binding affinity of CaMKIIa for $\mathrm{Ca}^{2+} / \mathrm{CaM}$ increases by Thr286 phosphorylation ${ }^{7}$. To examine to what degree Thr286 phosphorylation affects the decay kinetics of CaMKIIa-CaM interaction, we used a CaMKIIa ${ }^{\mathrm{T} 286 \mathrm{~A}}$ mutant sensor (Fig. 4). To minimize the effects of inter-subunit FRET between mEGFP-CaMKIIa ${ }^{\mathrm{T} 286 \mathrm{~A}}$ and mCherry-CaM bound to the adjacent endogenous wildtype CaMKII, we used hippocampal slices from Camk2a $a^{\mathrm{T} 286 \mathrm{~A}}$ knock-in mice. Thus, in this scheme, all the Thr286 residues in CaMKIIa subunits in a holoenzyme are mutated to Ala. We first compared the activation of the T286A mutant to that of mEGFP-CaMKIIa ${ }^{\mathrm{WT}}$ in response to a single glutamate uncaging pulse (Fig. 4a). We observed that the binding fraction increased to a level similar to that of wildtype, but the dissociation was faster by $\sim 3$ fold $(\tau=1.2 \pm 0.1 \mathrm{~s})$.

Next, we measured CaMKIIa ${ }^{\mathrm{T} 286 \mathrm{~A}}-\mathrm{CaM}$ association during sLTP induction (glutamate uncaging at $0.49 \mathrm{~Hz}$ ) (Fig. $4 \mathrm{~b}, \mathrm{c}$ ). Unlike the association of CaMKIIaWT with CaM, which plateaued after the first uncaging pulse, the association of CaMKIIa ${ }^{\text {T286A }}$ with CaM decayed after each uncaging pulse, showing a sawtooth-shaped pattern. However, the peak level of the binding fraction change of CaMKIIa ${ }^{\mathrm{T} 286 \mathrm{~A}}-\mathrm{CaM}$ was similar to that of CaMKIIa ${ }^{\text {WT }}$-CaM. The dissociation time constant of the CaM-CaMKIIa ${ }^{\mathrm{T} 286 \mathrm{~A}}$ interaction was obtained as $\tau=1.0 \pm$ $0.2 \mathrm{~s}\left(0.3 \pm 0.1\right.$ at $34-35^{\circ} \mathrm{C}$, Supplementary Fig. $\left.2 \mathrm{~b}, \mathrm{Q} 10=4.1\right)$.

We again observed a persistent component in the decay of

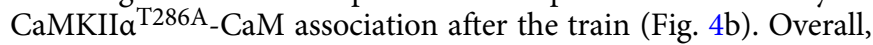
this component requires neither T286 phosphorylation nor CaM binding to the regulatory domain of CaMKIIa.

Inhibitory phosphorylations accelerate CaMKIIa inactivation. Next, we asked how the inhibitory phosphorylation at Thr305 and Thr306 may influence the $\mathrm{Ca}^{2+} / \mathrm{CaM}$ association during spine plasticity induction ${ }^{16,21}$. To do so, we mutated these phosphorylation sites from Threonine to Alanine and measured CaMKIIa $a^{\text {T305A/T306A }}$ association with $\mathrm{Ca}^{2+} / \mathrm{CaM}$ in response to glutamate uncaging. Following a single uncaging pulse, the binding fraction change of CaMKIIa $305 \mathrm{~A} / \mathrm{T} 306 \mathrm{~A}$ with $\mathrm{Ca}^{2+} / \mathrm{CaM}$ increased to a level similar to that of CaMKIIaWT but with a 
a

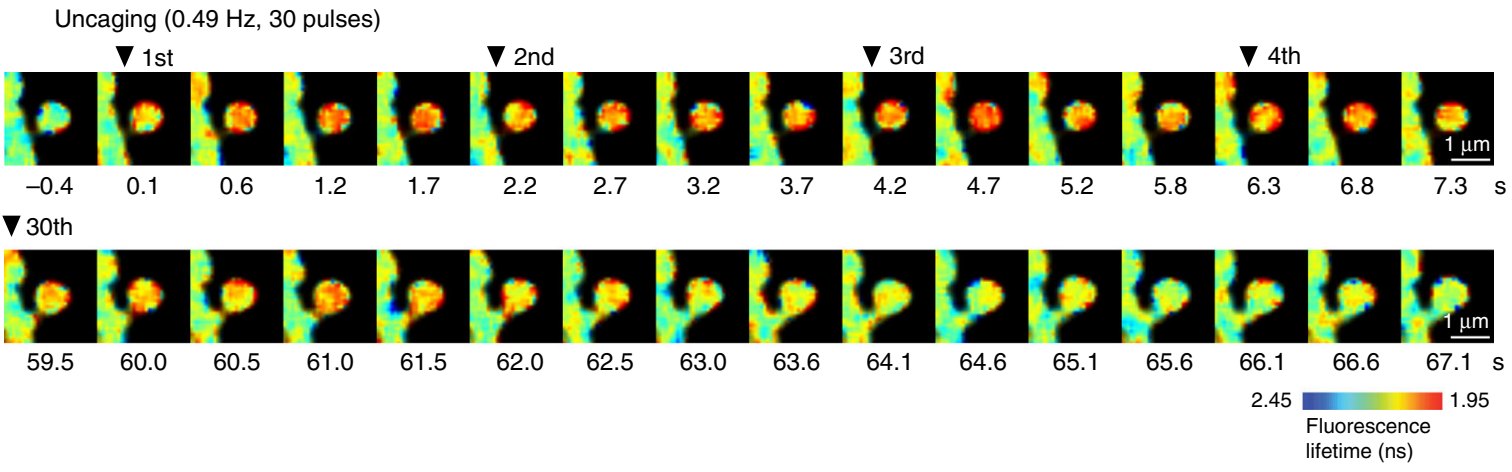

b
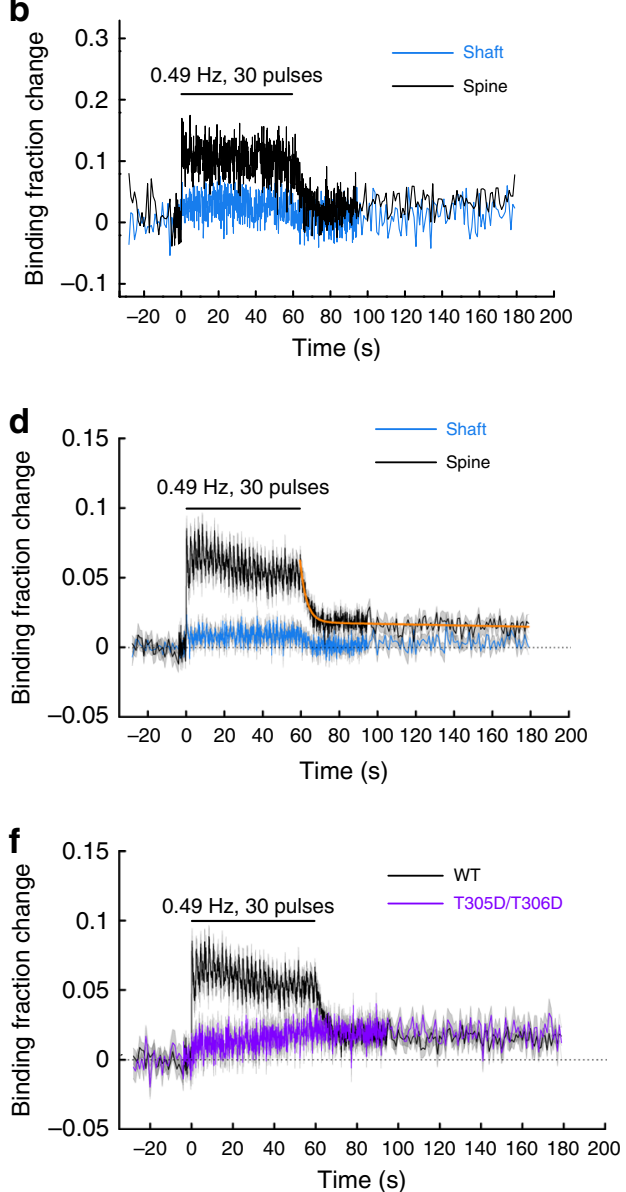
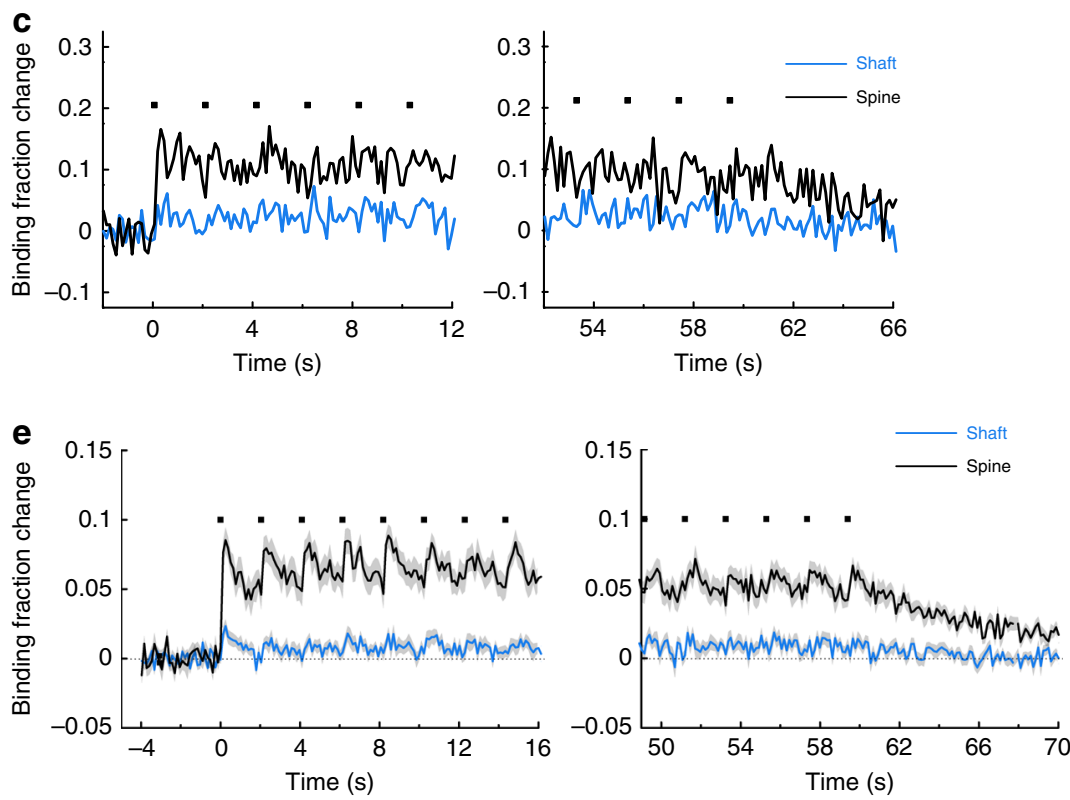

Fig. 2 CaMKIl $\alpha-\mathrm{CaM}$ association during sLTP induction. a Representative fluorescence lifetime images of CaMKIll $\alpha$-CaM association sensor during glutamate uncaging at $0.49 \mathrm{~Hz}$. Warmer colors indicate lower fluorescence lifetime, corresponding to a higher binding fraction of mCherry-CaM to mEGFPCaMKIl $\alpha$. Scale bar, $1 \mu \mathrm{m}$. b Time course of CaMKIl $\alpha$-CaM association in a stimulated spine (black) and nearby dendrite (blue). Analyzed from images in a. Black dots represent uncaging pulses. c Expanded view of the rising phase (left) and the decay phase (right) of $\mathbf{b}$. d Averaged change in CaMKIl $\alpha$-CaM association in stimulated spines (black) and nearby dendrite (blue) ( $n=27$ spines $/ 9$ neurons). The orange curve indicates the decay of binding fraction change obtained by curve fitting of a double-exponential function: $B(t)=B_{0}\left[P_{\text {fast }} \cdot \exp \left(-t / \tau_{\text {fast }}\right)+P_{\text {slow }} \cdot \exp \left(-t / \tau_{\text {slow }}\right)\right]$, where $B_{0}$ is the initial binding fraction change, $\tau_{\text {fast }}$ and $\tau_{\text {slow }}$ are the fast and slow decay time constants and $P_{\text {fast }}$ and $P_{\text {slow }}$ are the respective populations. The time constants are obtained as $\tau_{\text {fast }}$ $=3.2 \pm 0.6 \mathrm{~s}\left(P_{\text {fast }}=71 \%\right)$ and $\tau_{\text {slow }}=572 \pm 843 \mathrm{~s}\left(P_{\text {slow }}=29 \%\right)$. e Expanded view of the rising phase (left) and the decay phase (right) of $\mathbf{d}$. $\mathbf{f}$ Average time course of CaMKIl $\alpha-C a M$ association for a mutant mEGFP-CaMKIl $\alpha^{T 305 D / T 306 D}$ in which the Thr305 and Thr306 are mutated to aspartate. The mutation precludes $\mathrm{Ca}^{2+} / \mathrm{CaM}$ binding in the stimulated spine during glutamate uncaging at $0.49 \mathrm{~Hz}$ (purple; $n=34$ spines $/ 5$ neurons). The data for CaMKIlowT (black) are from c for the comparison. All data are shown in mean \pm sem, and sem of time constants is obtained by bootstrapping

slightly slower decay $(\tau=7.5 \pm 1.1 \mathrm{~s}$; Fig. 5a). During repetitive glutamate uncaging at $0.49 \mathrm{~Hz}$ (sLTP protocol), $\mathrm{Ca}^{2+} / \mathrm{CaM}$ binding to CaMKIIa ${ }^{\mathrm{T} 305 \mathrm{~A} / \mathrm{T} 306 \mathrm{~A}}$ increased to the level similar to that of CaMKIIaWT (Fig. 4b) and decayed with the time constant of $\tau=9.3 \pm 1.8 \mathrm{~s}$ (Fig. 5b, c), which was, again, slower than that of $\mathrm{CaMKIIa}^{\mathrm{WT}}(\tau \sim 3 \mathrm{~s})$. These results suggested that inhibitory phosphorylation at Thr305/Thr306 dynamically occurs during CaMKIIa activation, which inhibits the rebinding of $\mathrm{Ca}^{2+} / \mathrm{CaM}$ on CaMKIIa. However, preventing this regulation during the induction of sLTP (enhancing binding affinity to $\mathrm{Ca}^{2+} / \mathrm{CaM}$ ) did not result in a higher level of $\mathrm{Ca}^{2+} / \mathrm{CaM}$ binding. 

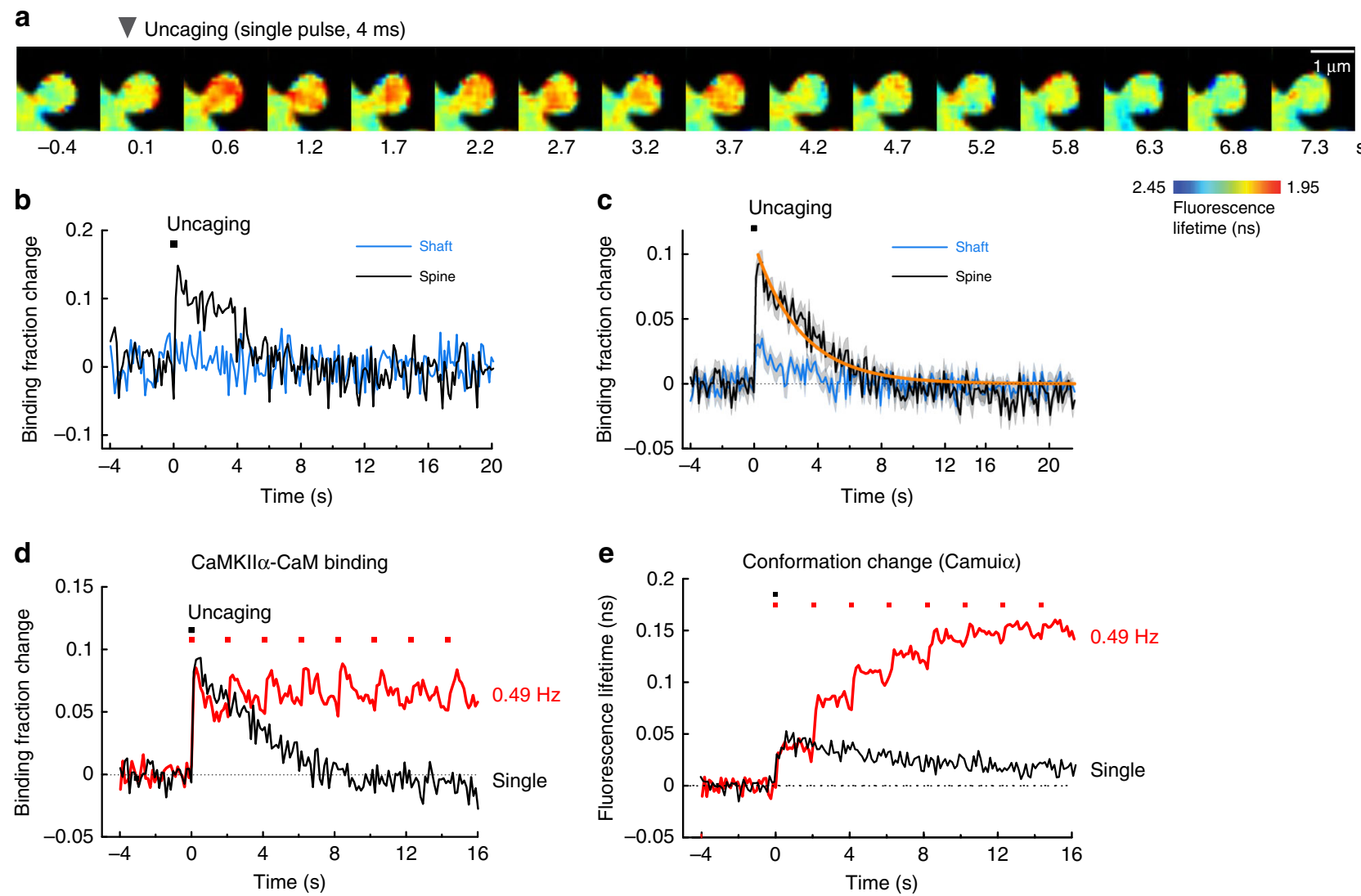

Fig. 3 CaMKIl $\alpha$-CaM association in response to a single glutamate uncaging pulse. a Representative fluorescence lifetime images of CaMKIl $\alpha$-CaM association in response to a single glutamate uncaging pulse. Warmer colors indicate lower fluorescence lifetime, corresponding to a higher binding fraction of mCherry-CaM to mEGFP-CaMKIl $\alpha$. Scale bar, $1 \mu \mathrm{m}$. b Time course of CaMKIl $\alpha$-CaM association in a stimulated spine (black) and nearby dendritic (blue). Inset is an expanded view of the rising phase. Black squares denote uncaging pulses. Analyzed from images in a. c Averaged changes in CaMKII $\alpha$-CaM association in spines and nearby dendrite $(n=28$ spines/4 neurons). The orange curve indicates the decay of binding fraction change obtained by curve fitting of an exponential function: $B(t)=B_{0} \exp (-t / \tau)$, where $B_{0}$ is the initial binding fraction change, $\tau$ is the dissociation time constant. The time constant is obtained as $\tau=2.9 \pm 0.3 \mathrm{~s}$. d Comparison of CaMKIl $\alpha$-CaM association in response to a single pulse (c) and to a train of glutamate uncaging (Fig. 2d, e). e CaMKIl $\alpha$ conformation change measured with Green-Camui $\alpha$ in response to a single pulse and a train of glutamate uncaging. Data from our previous publication ${ }^{10}$. All data are shown in mean \pm sem, and sem of time constants is obtained by bootstrapping

A kinetic model of CaMKIIa activation. Our results indicated that CaMKII-CaM association was rapidly activated by a single glutamate uncaging pulse, but did not show any integration over repetitive glutamate uncaging (Fig. 3d). In contrast, CaMKIIa activity measured with Camuia under similar conditions showed a high degree of integration ${ }^{10}$ (Figs. 3e, 6a). The plot assumes that the active population of CaMKIIa is equal to the fraction of CaMKIIa bound to $\mathrm{Ca}^{2+} / \mathrm{CaM}$ after the first pulse of glutamate. Since CaMKIIa autonomous activation is defined by active CaMKIIa without CaM binding, this population should be obtained by subtracting the fraction of CaMKIIa bound to CaM from CaMKIIa activity (cyan area, Fig. 6a). This suggests that CaMKIIa activity during sLTP is almost entirely supported by autonomous CaMKIIa activation.

To further clarify this point, we created a simple kinetic model of CaMKIIa (Fig. 6b, c). We constructed a set of rate equations to describe CaMKIIa biochemical reactions based on the proposed model (Table 1), and simulated the reaction in response to repetitive glutamate uncaging induced $\mathrm{Ca}^{2+}$ transients at $0.49 \mathrm{~Hz}$, our standard sLTP protocol (Fig. 6b, c). For $\mathrm{Ca}^{2+}$-CaM-CaMKIIa interaction, we used a model previously established based on biochemical experiments ${ }^{22}$ (Table 1). When two adjacent subunits are activated, CaMKIIa subunit $(\mathrm{K})$ undergoes phosphorylation $(\mathrm{P})$. We assume that the rate of phosphorylation $\left(k_{1}\right)$ is proportional to the chance that the adjacent subunit is active: the fraction of CaM-bound, unphosphorylated form (KCaM), plus CaM-bound, phosphorylated form (PCaM), plus phosphorylated subunit ( $\mathrm{P}$ and $\mathrm{P}_{2}$; see below for the explanation of the $\mathrm{P}_{2}$ state). The maximum rate has been reported to be $6.3 \mathrm{~s}^{-1}$ (ref. ${ }^{23}$ ), but we found that a two-fold higher value $\left(12.6 \mathrm{~s}^{-1}\right)$ fits our data better. Following previous kinetic models ${ }^{22,23}$, we assume that $\mathrm{CaM}$ rebinding to the $\mathrm{P}$ state $(\mathrm{P} \rightarrow \mathrm{PCaM})$ and dephosphorylation while the subunit bound to $\mathrm{CaM}$ (PCaM $\rightarrow$ $\mathrm{KCaM}$ ) do not occur. The rate of CaM dissociation from PCaM $\left(k_{2}\right)$ was measured to be $1 / 3 \mathrm{~s}^{-1}$ (or time constant of $3 \mathrm{~s}$ ) in this study (Fig. 1), and the rate of dephosphorylation of CaMKIIa $\left(k_{3}\right)$ has been previously measured to be $1 / 6 \mathrm{~s}^{-1}$ (or time constant of $6 \mathrm{~s}$; Chang et al.). In addition, we assume that the persistent component of the FRET signal of CaMKIIa-CaM association is not related to the activation of CaMKIIa, as it is sensitive neither to T286A mutation nor to T305D/T306D mutations. However, the slow component of CaMKIIa activation measured with Camuia (time constant $\sim 1 \mathrm{~min}$ ) depends on T286 phosphorylation, and thus likely represents the autophosphorylation state of $\mathrm{CaMKII}^{10}$. To explain this component of decay in CaMKIIa activation, we included a slow phosphorylation state $\left(\mathrm{P}_{2}\right)$ with the time constant of $1 \mathrm{~min}\left(k_{5}=1 / 60 \mathrm{~s}^{-1}\right)$. The fraction of slow component of CaMKIIa activation ( $25 \%$; Chang et al.) can be approximated by the ratio of $k_{3}$ and $k_{4}$, we set $k_{3}$ to be $0.25 k_{3}$ $\left(k_{4}=0.25 / 6 \mathrm{~s}^{-1}\right)$. Overall, we obtained most of the kinetic 

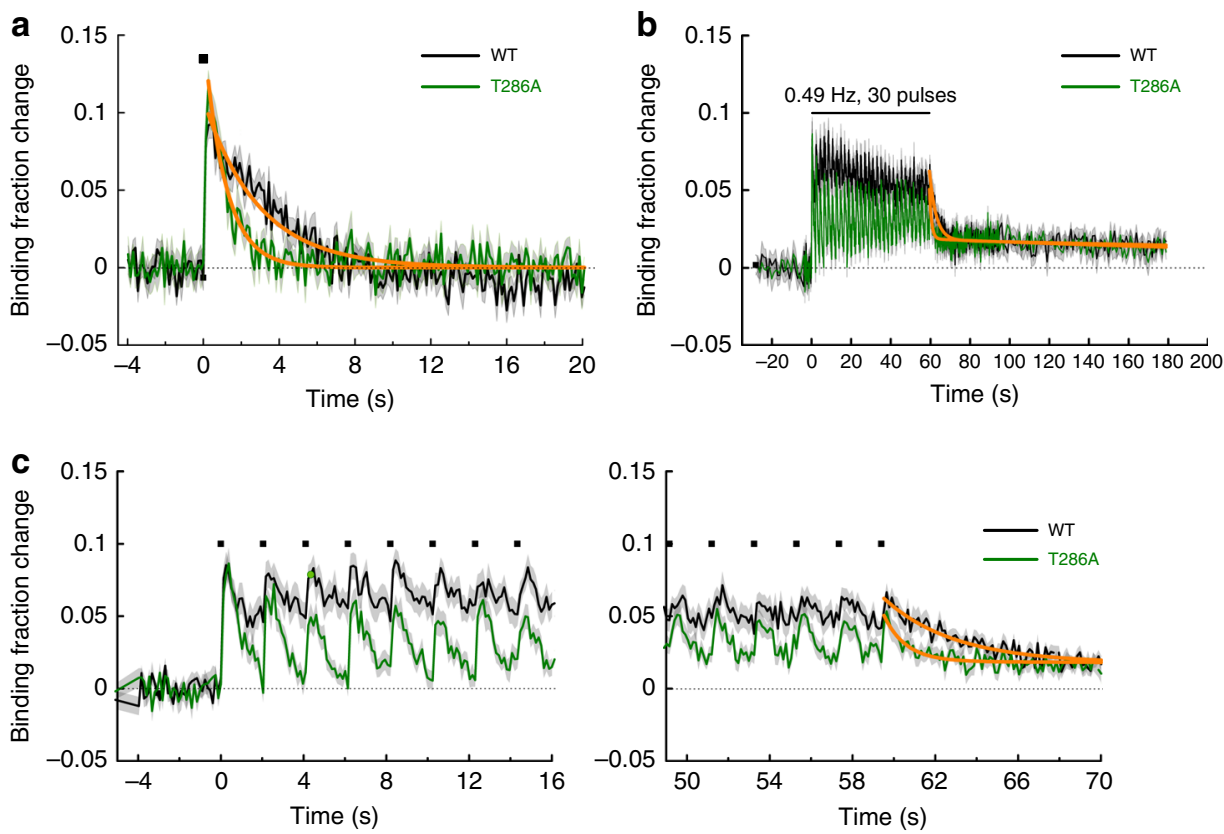

Fig. 4 CaMKII $\alpha^{T 286 \mathrm{~A}}-\mathrm{CaM}$ association during SLTP induction. a Averaged change in CaMKIl $\alpha^{\mathrm{T} 286 \mathrm{~A}}-\mathrm{CaM}$ association in a stimulated spine (green; $n=$ 18 spines/4 neurons) in response to a single glutamate uncaging pulse (black square). The orange curve on CaMKIl ${ }^{\top 286 \mathrm{~A}}$ is obtained by curve fitting of an exponential function: $B(t)=B_{0} \cdot e^{-t / \tau}$. The dissociation time constant is obtained as $\tau=1.2 \pm 0.1 \mathrm{~s}$. The data and fitted curve for CaMKIl $\mathrm{WT}$ are from Fig. $3 \mathrm{c}$ for the comparison. $\mathbf{b}$ Averaged change in CaMKIl ${ }^{\top 286 \mathrm{~A}}-\mathrm{CaM}$ association in stimulated spines (green; $n=24$ spines/7 neurons) during glutamate uncaging at $0.49 \mathrm{~Hz}$. The orange curve indicates the decay of binding fraction change obtained by curve fitting of a double-exponential function: $B(t)=B_{0}$ $\left[P_{\text {fast }} \cdot \exp \left(-t / \tau_{\text {fast }}\right)+P_{\text {slow }} \cdot \exp \left(-t / \tau_{\text {slow }}\right)\right]$. The time constants for CaMKIl $\alpha^{\top 286 \mathrm{~A}}$ are obtained as $\tau_{\text {fast }}=1.0 \pm 0.2 \mathrm{~s}\left(P_{\text {fast }}=63 \%\right)$ and $\tau_{\text {slow }}=356 \pm 221 \mathrm{~s}$ $\left(P_{\text {slow }}=37 \%\right)$. The data and fitted curve for CaMKIl $\alpha$ WT (black) are from Fig. $2 \mathrm{~d}$ for the comparison. $\mathbf{c}$ Expanded view of the initial phase (left) and the late (right) phase of plot in $\mathbf{b}$. All data are shown in mean \pm sem, and sem of time constants is obtained by bootstrapping
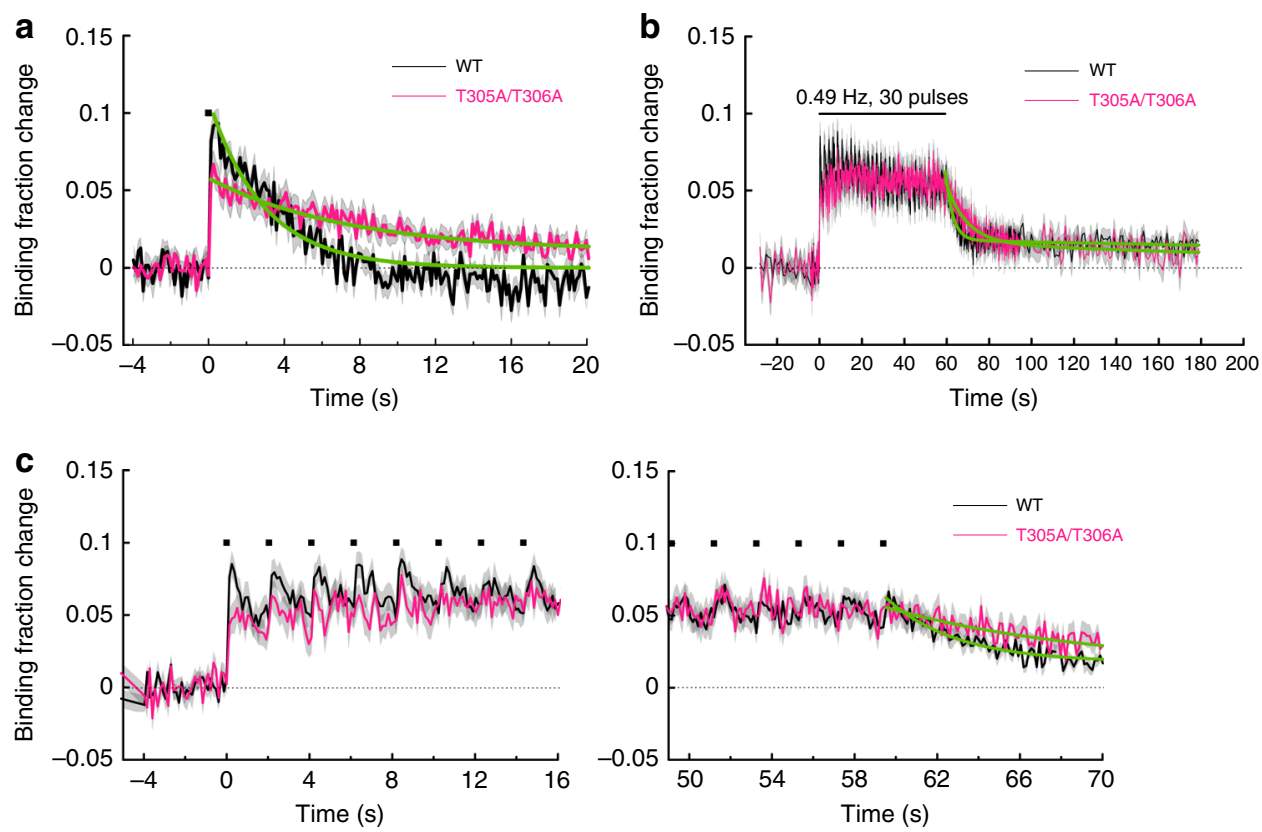

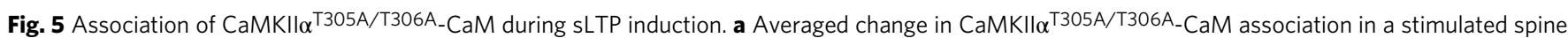
(magenta; $n=34$ spines/ 6 neurons) in response to a single glutamate uncaging pulse (black square). The green curve on CaMKIl ${ }^{\top 305 A / T 306 \mathrm{~A}}$ is obtained by curve fitting of an exponential function: $B(t)=B_{0} \exp (-t / \tau)$. The dissociation time constant is obtained as $\tau=7.5 \pm 1.1 \mathrm{~s}$. Inset is a expanded view. The

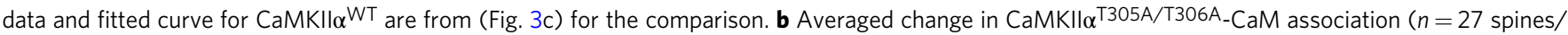
8 neurons) during glutamate uncaging at $0.49 \mathrm{~Hz}$. The green curve indicates the decay of binding fraction change obtained by curve fitting of a doubleexponential function: $B(t)=B_{0}\left[P_{\text {fast }} \cdot \exp \left(-t / \tau_{\text {fast }}\right)+P_{\text {slow }} \cdot \exp \left(-t / \tau_{\text {slow }}\right)\right]$. The time constants are obtained as $\tau_{\text {fast }}=9.3 \pm 1.8 \mathrm{~s}\left(P_{\text {fast }}=71 \%\right)$ and $\tau_{\text {slow }}=249$ $\pm 229 \mathrm{~s}\left(P_{\text {slow }}=29 \%\right)$. The data and fitted curve for CaMKIl $\alpha$ WT are from (Fig. 2d) for the comparison. c Expanded view of the initial phase (left) and the late phase (right) in $\mathbf{b}$ 
a

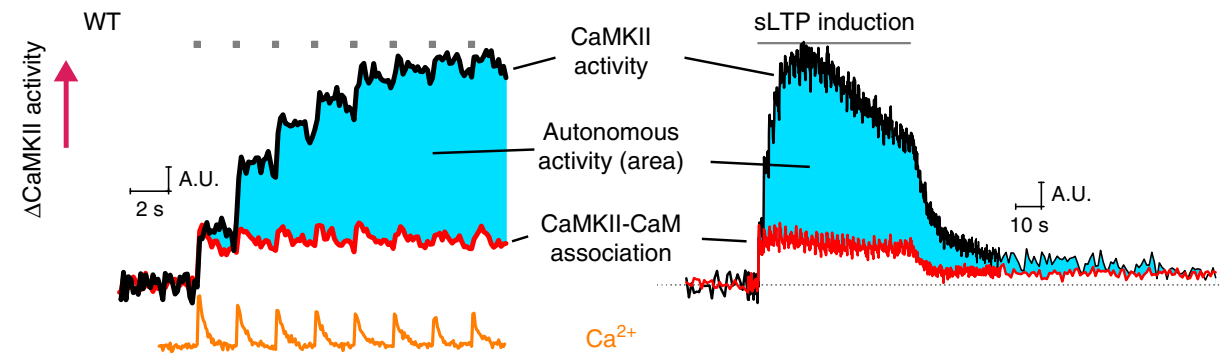

b
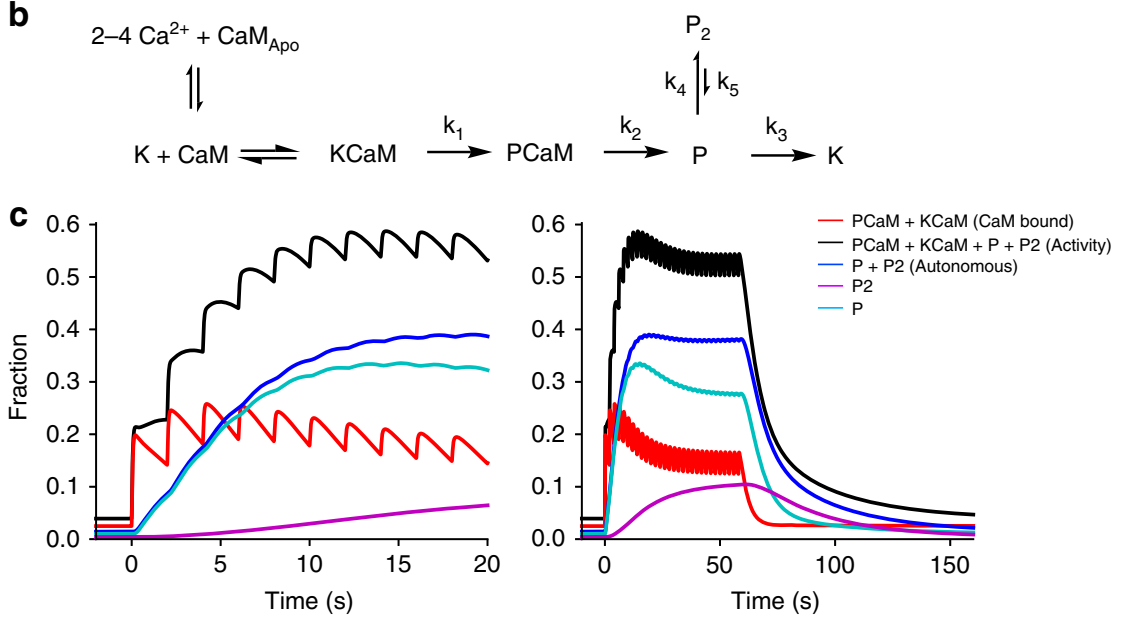

Fig. 6 Simulated CaMKIl $\alpha$ activation during spine plasticity induction. a The comparison of CaMKIl $\alpha$ activity measured with Green-Camui $\alpha$ (data from ref. ${ }^{10}$ ), $\mathrm{Ca}^{2+}$ measured with Fluo-4FF (data from ref. ${ }^{10}$ ), and CaMKIl $\alpha$-CaM association measured in this study. Autonomous activity is the subtraction of CaMKIl $\alpha$-CaM association from CaMKIl $\alpha$ activation (cyan). The first time point right after uncaging is matched for CaMKIl $\alpha$-CaM and CaMKIl $\alpha$ activity, assuming that there is no autonomous CaMKIl $\alpha$ at the time point. $\mathbf{b}$ Reaction scheme of CaMKIl $\alpha$ activation. $\mathrm{K}$ is the inactive state of CaMKIl $\alpha$ (closed form), $\mathrm{CaM}_{\mathrm{Apo}}$ is the inactive form of calmodulin without bound $\mathrm{Ca}^{2+}, \mathrm{CaM}$ is the active form of calmodulin with 2-4 bound $\mathrm{Ca}^{2+}$ ions, $\mathrm{P}$ and $\mathrm{P}_{2}$ are the two different states of Thr286-phosphorylated CaMKIl $\alpha$. c Simulated CaMKIl $\alpha$ activation based on the proposed reaction scheme. Black:

concentration of total active CaMKIl $\alpha\left(K C a M+P C a M+P+P_{2}\right)$. Red: concentration of KCaM and PCaM. Green: concentration of Thr286-phosphorylated CaMKIl $\alpha\left(P+P_{2}\right)$. Light blue: concentration of $P$ state of CaMKIl $\alpha$. Navy: concentration of $P_{2}$ state of CaMKIl $\alpha$

parameters necessary for simulating the reaction (parameters for $\mathrm{CaM}$ association and $k_{1}-k_{5}$ in Fig. $6 \mathrm{~b}$ ) from this and previous studies $^{10,22}$ (Table 1).

This reaction scheme recapitulates several key features of CaMKIIa activation and CaM-CaMKIIa binding in single dendritic spines: (1) decay kinetics of CaMKIIa activation with two time constants ${ }^{10}$, (2) integration of CaMKIIa activation in response to each pulse ${ }^{10}$, (3) no accumulation of CaMKIIa-CaM interaction during repetitive $\mathrm{Ca}^{2+}$ pulses, (4) decay of CaMKIIa activity in response to a single pulse $(\sim 10 \mathrm{~s})$, which is longer than that following a train of pulses $(\sim 6 s)^{10}$, (5) time course of CaM binding to T286A mutant, simulated by removing the effects of phosphorylation (setting $k_{1}$ to 0 ). The model shows that autonomous CaMKIIa activation $\left(\mathrm{P}+\mathrm{P}_{2}\right)$ increases over time, and becomes the dominant population after $\sim 3-4$ uncaging pulses.

Previously our and other groups examined the effects of Thr286 dephosphorylation on CaMKIIa activity using Camuia sensor with T286D mutation or wildtype Camuia sensor in the presence of phosphatase inhibitor ${ }^{10,24}$. These studies showed a high basal level of CaMKIIa activity before glutamate uncaging, consistent with this study that T286 phosphorylation accounts for most of CaMKIIa activation. Interestingly, both studies showed that there exists a smaller, rapid increase of active CaMKIIa which decays rapidly after cessation of glutamate uncaging. Since the phosphorylation state of T286 is constantly in "on" state under this condition, this rapid activation must be due to the association/ dissociation of $\mathrm{Ca}^{2+} / \mathrm{CaM}$ from CaMKIIa ${ }^{\mathrm{T} 286 \mathrm{D}}$.
To simulate the activation of CaMKIIa ${ }^{\text {T286D }}$, we slightly modified the above model. First, we allowed phosphorylated CaMKIIa binds to CaM (P $\rightarrow$ PCaM) with $10 \%$ of the association rate of non-phosphorylated $\mathrm{K}-\mathrm{CaM}$ association $(\mathrm{K} \rightarrow \mathrm{KCaM})$. Second, we assumed that CaMKIIa activity in the autonomous state $\left(\mathrm{P}\right.$ or $\left.\mathrm{P}_{2}\right)$ is $60 \%$ as high as that when binding with $\mathrm{CaM}$ (KCaM or PCaM), as measured previously with FRET sensors ${ }^{6,17}$ and substrate phosphorylation ${ }^{25}$ (Fig. 7a). This model produced a time course of CaMKIIa ${ }^{\mathrm{WT}}$ activation similar to that produced by the original model (Fig. 7b). Importantly, when we set dephosphorylation rate to zero to simulate T286D mutation, we recapitulated all above features of CaMKIIa ${ }^{\mathrm{T} 286 \mathrm{D}}$ activity ${ }^{10,24}$ (Fig. $7 \mathrm{~b}, \mathrm{c}$ ), including high basal activity and a rapid activation and inactivation due to CaM binding and unbinding, respectively. The same model also reproduced the activity profile of T286A mutation (set the rate of phosphorylation to 0), showing smaller basal activity, smaller activation, and faster decay ${ }^{10}$ (Fig. 7b, c).

Finally, we examined how this model predicts CaMKIIa activation during spike-timing-dependent plasticity (STDP), in which LTP can be induced by pairing synaptic stimulation with back-propagating action potentials (bAP) with slight delay ${ }^{26}$. We assumed that bAPs produce $\mathrm{Ca}^{2+}$ transient with the peak concentration of $0.8 \mu \mathrm{M}$ and the decay time constant of $20 \mathrm{~ms}^{27,28}$. When paired with synaptic release at the synapse, $\sim 3$ times more $\mathrm{Ca}^{2+}$ is produced ${ }^{29}$. In this model, bAPs alone produced little CaMKIIa activation (Fig. 7d). However, when paired with synaptic activity, the stimulation activated CaMKIIa to a higher level, reaching the level similar to that produced 


\section{Table 1 List of parameters used for simulation}

\begin{tabular}{|c|c|c|c|}
\hline Name & Meaning & Value (Rate constant or concentration) & Note \\
\hline CaM & Calmodulin & & \multirow{30}{*}{$\begin{array}{l}\text { Values from Pepke et al. }{ }^{22} \\
\text { Coarse grained model by Pepke et al. }{ }^{22} \\
\text { for R1-R24 }\end{array}$} \\
\hline $\mathrm{CaM}_{\text {Apo }}$ & Calmodulin without bound $\mathrm{Ca}^{2+}$ & & \\
\hline $\mathrm{Ca}(\mathrm{n}) \mathrm{CaM}-\mathrm{C}$ & Calmodulin binding $\mathrm{n} \mathrm{Ca}{ }^{2+}$ on its $\mathrm{C}$-lobe & & \\
\hline $\mathrm{Ca}(\mathrm{n}) \mathrm{CaM}-\mathrm{N}$ & Calmodulin binding $\mathrm{n} \mathrm{Ca}{ }^{2+}$ on its $\mathrm{N}$-lobe & & \\
\hline $\mathrm{Ca} 4 \mathrm{CaM}$ & Calmodulin binding $4 \mathrm{Ca}^{2+}$ & & \\
\hline $\mathrm{KCaM}$ & $\begin{array}{l}\text { CaMKIl } \alpha \text { without T286 phosphorylation } \\
\text { bound to CaM }\end{array}$ & & \\
\hline K & CaMKIl $\alpha$ without T286 phosphorylation & & \\
\hline $\mathrm{P}$ & CaMKII $\alpha$ with Thr286 phosphorylation & & \\
\hline$P_{2}$ & $\begin{array}{l}\text { A different form of CaMKIl } \alpha \text { with Thr286 } \\
\text { phosphorylation }\end{array}$ & & \\
\hline KCaM & $\mathrm{K}$ associated with $\mathrm{CaM}$ & & \\
\hline PCaM & $\mathrm{P}$ associated with CaM & & \\
\hline $\mathrm{F}$ & $\begin{array}{l}\text { Fraction of active CaMKII subunits, } \\
\mathrm{KCaM}+\mathrm{PCaM}+\mathrm{P}+\mathrm{P}_{2}\end{array}$ & & \\
\hline CaM1C $_{\text {on }}$ & $\mathrm{Ca}^{2+}+\mathrm{CaM}_{\mathrm{Apo}} \rightarrow \mathrm{CaCaM}-\mathrm{C}$ & $5 \times 10^{6} \mathrm{M}^{-1} \mathrm{~s}^{-1}$ & \\
\hline $\mathrm{CaM1C}_{\text {off }}$ & $\mathrm{CaCaM}-\mathrm{C} \rightarrow \mathrm{Ca}^{2+}+\mathrm{CaM}_{\mathrm{Apo}}$ & $50 s^{-1}$ & \\
\hline $\mathrm{CaM} 2 \mathrm{C}_{\text {on }}$ & $\mathrm{Ca}^{2+}+\mathrm{CaCaM}-\mathrm{C} \rightarrow \mathrm{Ca} 2 \mathrm{CaM}-\mathrm{C}$ & $10 \times 10^{6} \mathrm{M}^{-1} \mathrm{~s}^{-1}$ & \\
\hline $\mathrm{CaM} 2 \mathrm{C}_{\text {off }}$ & $\mathrm{Ca} 2 \mathrm{CaM}-\mathrm{C} \rightarrow \mathrm{Ca}^{2+}+\mathrm{CaCaM}-\mathrm{C}$ & $10 s^{-1}$ & \\
\hline CaM1N on & $\mathrm{Ca}^{2+}+\mathrm{CaM}_{\mathrm{Apo}} \rightarrow \mathrm{CaCaM}-\mathrm{N}$ & $100 \times 10^{6} \mathrm{M}^{-1} \mathrm{~s}^{-1}$ & \\
\hline CaM1N off & $\mathrm{CaCaM}-\mathrm{N} \rightarrow \mathrm{Ca}^{2+}+\mathrm{CaM}-\mathrm{N}$ & $2 \times 10^{3} s$ & \\
\hline $\mathrm{CaM} 2 \mathrm{~N}_{\text {on }}$ & $\mathrm{Ca}^{2+}+\mathrm{CaCaM}-\mathrm{N} \rightarrow \mathrm{Ca} 2 \mathrm{CaM}-\mathrm{N}$ & $200 \times 10^{6} \mathrm{M}^{-1} \mathrm{~S}^{-1}$ & \\
\hline $\mathrm{CaM} 2 \mathrm{~N}_{\text {off }}$ & $\mathrm{Ca} 2 \mathrm{CaM}-\mathrm{N} \rightarrow \mathrm{Ca}^{2+}+\mathrm{Ca} 2 \mathrm{CaM}$ & $500 s^{-1}$ & \\
\hline $\mathrm{KCaM}_{1} \mathrm{C}_{\text {on }}$ & $\mathrm{Ca}^{2+}+\mathrm{KCaM}_{\mathrm{Apo}} \rightarrow \mathrm{KCaCaM}-\mathrm{C}$ & $44 \times 10^{6} \mathrm{M}^{-1} \mathrm{~s}^{-1}$ & \\
\hline $\mathrm{KCaM} 1 \mathrm{C}_{\text {off }}$ & $\mathrm{KCaCaM}-\mathrm{C} \rightarrow \mathrm{Ca}^{2+}+\mathrm{KCaM}_{\text {Apo }}$ & $33 s^{-1}$ & \\
\hline $\mathrm{KCaM} 2 \mathrm{C}_{\text {on }}$ & $\mathrm{Ca}^{2}++\mathrm{KCaCaM}-\mathrm{C} \rightarrow \mathrm{KCa} 2 \mathrm{CaM}-\mathrm{C}$ & $44 \times 10^{6} \mathrm{M}^{-1} \mathrm{~s}^{-1}$ & \\
\hline $\mathrm{KCaM} 2 \mathrm{C}_{\text {off }}$ & $\mathrm{KCa} 2 \mathrm{CaM}-\mathrm{C} \rightarrow \mathrm{Ca}^{2}++\mathrm{KCaCaM}-\mathrm{C}$ & $0.8 s^{-1}$ & \\
\hline $\mathrm{KCaM}_{1} \mathrm{~N}_{\text {on }}$ & $\mathrm{Ca}^{2+}+\mathrm{KCaM}_{\mathrm{Apo}} \rightarrow \mathrm{KCaCaM}-\mathrm{N}$ & $76 \times 10^{6} \mathrm{M}^{-1} \mathrm{~s}^{-1}$ & \\
\hline $\mathrm{KCaM} \mathrm{N}_{\text {off }}$ & $\mathrm{KCaCaM}-\mathrm{N} \rightarrow \mathrm{Ca}^{2+}+\mathrm{KCaM}_{\text {Apo }}$ & $300 s^{-1}$ & \\
\hline $\mathrm{KCaM} 2 \mathrm{~N}_{\text {on }}$ & $\mathrm{Ca}^{2+}+\mathrm{KCaCaM}-\mathrm{N} \rightarrow \mathrm{KCa} 2 \mathrm{CaM}-\mathrm{N}$ & $76 \times 10^{6} \mathrm{M}^{-1} \mathrm{~s}^{-1}$ & \\
\hline $\mathrm{KCaM} 2 \mathrm{~N}_{\text {off }}$ & $\mathrm{KCa} 2 \mathrm{CaM}-\mathrm{N} \rightarrow \mathrm{Ca}^{2+}+\mathrm{KCaCaM}-\mathrm{N}$ & $20 \underset{\mathrm{CaM1}}{\mathrm{s}_{o n}^{-1}} \cdot \mathrm{CaM}_{2} \mathrm{C}_{o n}$ & \\
\hline R1 & $2 \mathrm{Ca}^{2+}+\mathrm{CaM}_{\mathrm{Apo}} \rightarrow \mathrm{Ca} 2 \mathrm{CaM}-\mathrm{C}$ & $\frac{\mathrm{CaM1} \mathrm{C}_{\text {on }} \cdot \mathrm{CaM2} \mathrm{C}_{\text {on }}}{\mathrm{CaM1C}_{\text {off }}+\mathrm{CaM} 2 \mathrm{C}_{\text {on }}\left[\mathrm{Ca}^{2+}\right]}$ & \\
\hline R2 & $\mathrm{Ca} 2 \mathrm{CaM}-\mathrm{C} \rightarrow 2 \mathrm{Ca}^{2+}+\mathrm{CaM}_{\mathrm{Apo}}$ & $\frac{\mathrm{CaM} 1 \mathrm{C}_{\text {off }} \mathrm{CaM} 2 \mathrm{C}_{\text {off }}}{\mathrm{CaM1C_{ \text {off } }}+\mathrm{CaM} 2 \mathrm{C}_{\text {on }}\left[\mathrm{Ca}^{2+}\right]}$ & \\
\hline R3 & $2 \mathrm{Ca}^{2+}+\mathrm{CaM}_{\mathrm{Apo}} \rightarrow \mathrm{Ca} 2 \mathrm{CaM}-\mathrm{N}$ & $\frac{\mathrm{CaM} 1 \mathrm{~N}_{\text {on }} \cdot \mathrm{CaM} 2 \mathrm{~N}_{\text {on }}}{\mathrm{CaM1N_{ \text {off } }}+\mathrm{CaM} 2 \mathrm{~N}_{\text {on }}\left[\mathrm{Ca}^{2+}\right]}$ & \\
\hline R4 & $\mathrm{Ca} 2 \mathrm{CaM}-\mathrm{N} \rightarrow 2 \mathrm{Ca}^{2+}+\mathrm{CaM}_{\mathrm{Apo}}$ & $\frac{\mathrm{CaM} 1 \mathrm{~N}_{\text {off }} \cdot \mathrm{CaM} 2 \mathrm{~N}_{\text {off }}}{\mathrm{CaM1N_{ \text {off } }}+\mathrm{CaM} 2 \mathrm{~N}_{\text {on }}\left[\mathrm{Ca}^{2+}\right]}$ & \\
\hline R5 & $2 \mathrm{Ca}^{2+}+\mathrm{Ca} 2 \mathrm{CaM}-\mathrm{C} \rightarrow \mathrm{Ca} 4 \mathrm{CaM}$ & Same as R3 & \\
\hline R6 & $\mathrm{Ca} 4 \mathrm{CaM} \rightarrow 2 \mathrm{Ca}^{2+}+\mathrm{Ca} 2 \mathrm{CaM}-\mathrm{C}$ & Same as R4 & \\
\hline R7 & $2 \mathrm{Ca}^{2+}+\mathrm{Ca} 2 \mathrm{CaM}-\mathrm{N} \rightarrow \mathrm{Ca} 4 \mathrm{CaM}$ & Same as R1 & \\
\hline R8 & $\mathrm{Ca} 4 \mathrm{CaM} \rightarrow 2 \mathrm{Ca}^{2+}+\mathrm{Ca} 2 \mathrm{CaM}-\mathrm{N}$ & Same as R2 & \\
\hline R9 & $2 \mathrm{Ca}^{2+}+\mathrm{KCaM}_{\mathrm{Apo}} \rightarrow \mathrm{KCa} 2 \mathrm{CaM}-\mathrm{C}$ & $\frac{K C a M 1 C_{\text {on }} \cdot K C a M 2 C_{\text {on }}}{K^{2+} \mathrm{KaM}_{1} \mathrm{C}_{\text {off }}+\mathrm{KCaM} 2 \mathrm{C}_{\text {on }}\left[\mathrm{Ca}^{2+}\right]}$ & \\
\hline R10 & $\mathrm{KCa} 2 \mathrm{CaM}-\mathrm{C} \rightarrow 2 \mathrm{Ca}^{2+}+\mathrm{KCaM}_{\mathrm{Apo}}$ & $\frac{K C a M 1 C_{\text {off }} \cdot K C a M 2 C_{\text {off }}}{K C a M 1 C_{\text {off }}+K C a M 2 C_{\text {on }}\left[\mathrm{Ca}^{2+}\right]}$ & \\
\hline R11 & $2 \mathrm{Ca}^{2+}+\mathrm{KCaM}_{\mathrm{Apo}} \rightarrow \mathrm{KCa} 2 \mathrm{CaM}-\mathrm{N}$ & $\frac{K C a M 1 N_{\text {on }} \cdot K C a M 2 N_{\text {on }}}{K C a M 1 N_{\text {off }}+K C a M 2 N_{\text {on }}\left[\mathrm{Ca}^{2+}\right]}$ & \\
\hline R12 & $\mathrm{KCa} 2 \mathrm{CaM}-\mathrm{N} \rightarrow 2 \mathrm{Ca}^{2+}+\mathrm{KCaM}_{\mathrm{Apo}}$ & $\frac{K C a M 1 N_{\text {off }} \cdot K C a M 2 N_{\text {off }}}{K C a M 1 N_{\text {off }}+K C a M 2 N_{\text {on }}\left[\mathrm{Ca}^{2+}\right]}$ & \\
\hline R13 & $2 \mathrm{Ca}^{2+}+\mathrm{KCa} 2 \mathrm{CaM}-\mathrm{C} \rightarrow \mathrm{KCa} 4 \mathrm{CaM}$ & Same as R11 & \\
\hline R14 & $\mathrm{KCa} 4 \mathrm{CaM} \rightarrow 2 \mathrm{Ca}^{2+}+\mathrm{KCa} 2 \mathrm{CaM}-\mathrm{C}$ & Same as R12 & \\
\hline R15 & $2 \mathrm{Ca}^{2+}+\mathrm{KCa} 2 \mathrm{CaM}-\mathrm{N} \rightarrow \mathrm{KCa} 4 \mathrm{CaM}$ & Same as R9 & \\
\hline R16 & $\mathrm{KCa} 4 \mathrm{CaM} \rightarrow 2 \mathrm{Ca}^{2+}+\mathrm{KCa} 2 \mathrm{CaM}-\mathrm{N}$ & Same as R10 & \\
\hline R17 & $\mathrm{K}+\mathrm{CaM}_{\text {Apo }} \rightarrow \mathrm{KCaM}_{\text {Apo }}$ & $3.8 \times 10^{3} \mathrm{M}^{-1} \mathrm{~s}^{-1}$ & \\
\hline R18 & $\mathrm{KCaM}_{\mathrm{Apo}} \rightarrow \mathrm{K}+\mathrm{CaM}_{\mathrm{Apo}}$ & $5.5 \mathrm{~s}^{-1}$ & \\
\hline R19 & $\mathrm{K}+\mathrm{Ca} 2 \mathrm{CaM}-\mathrm{C} \rightarrow \mathrm{KCa} 2 \mathrm{CaM}-\mathrm{C}$ & $0.92 \times 10^{3} \mathrm{M}^{-1} \mathrm{~s}^{-1}$ & \\
\hline R20 & $\mathrm{KCa} 2 \mathrm{CaM}-\mathrm{C} \rightarrow \mathrm{K}+\mathrm{Ca} 2 \mathrm{CaM}-\mathrm{C}$ & $6.8 s^{-1}$ & \\
\hline R21 & $\mathrm{K}+\mathrm{Ca} 2 \mathrm{CaM}-\mathrm{N} \rightarrow \mathrm{KCa} 2 \mathrm{CaM}-\mathrm{N}$ & $0.12 \times 10^{3} \mathrm{M}^{-1} \mathrm{~S}^{-1}$ & \\
\hline R22 & $\mathrm{KCa} 2 \mathrm{CaM}-\mathrm{N} \rightarrow \mathrm{K}+\mathrm{Ca} 2 \mathrm{CaM}-\mathrm{N}$ & $1.7 \mathrm{~s}^{-1}$ & \\
\hline R23 & $\mathrm{K}+\mathrm{Ca} 4 \mathrm{CaM} \rightarrow \mathrm{KCa} 4 \mathrm{CaM}$ & $30 \times 10^{3} \mathrm{M}^{-1} \mathrm{~s}^{-1}$ & \\
\hline R24 & $\mathrm{KCa} 4 \mathrm{CaM} \rightarrow \mathrm{K}+\mathrm{Ca} 4 \mathrm{CaM}$ & $1.5 s^{-1}$ & \\
\hline R25 & $\mathrm{KCaM} \rightarrow \mathrm{PCaM}$ & $k_{1}: F \times 12.6 \mathrm{~s}^{-1}$ & $\begin{array}{l}6.3 \text { according to Lucic et al. }{ }^{23} \mathrm{~F} \text { is the fraction } \\
\text { of active CaMKII subunits }\end{array}$ \\
\hline R26 & $\mathrm{PCaM} \rightarrow \mathrm{P}+\mathrm{CaM}$ & $k_{2}: 0.33 s^{-1}$ & Decay of $\mathrm{Ca}^{2+}-$ CaMKII association, $3 \mathrm{~s}$. \\
\hline R27 & $\mathrm{P} \rightarrow \mathrm{P} 2$ & $k_{4}: 0.041 \mathrm{~s}^{-1}$ & $k_{3} / k_{4}=1 / 4$ : the fraction of slow component \\
\hline R28 & $\mathrm{P} 2 \rightarrow \mathrm{P}$ & $k_{5}: 0.017 \mathrm{~s}^{-1}$ & Slow decay of CaMKII: 60 s Chang et al. ${ }^{10}$ \\
\hline R29 & $\mathrm{P} \rightarrow \mathrm{K}$ & $k_{3}: 0.17 \mathrm{~s}^{-1}$ & Fast decay of CaMKII activity: 6 s (ref. 10) \\
\hline R30 - R33 & $\begin{array}{l}\text { Same as R17, R19, R21, R23, with K } \\
\text { replaced by P }\end{array}$ & $\begin{array}{l}0.1 \times \text { R17, R19, R21, R23 for the model in Fig. S3a and } \\
0 \text { for the model in Fig. } 6 \text { a }\end{array}$ & $\begin{array}{l}\mathrm{Ca}^{2+} / \mathrm{CaM} \text { binding to phosphorylated } \\
\text { CaMKII (P) }\end{array}$ \\
\hline R34 - R41 & Same as R9 - R16, with $K$ replaced by $P$ & Same as R9 - R16. & $\mathrm{Ca}^{2+}$ binding to $\mathrm{CaM}$ on $\mathrm{P}$ \\
\hline$\left[\mathrm{Ca}^{2+}\right]_{\text {peak }}$ & Peak $\left[\mathrm{Ca}^{2+}\right]$ & $\begin{array}{l}4 \mu \mathrm{M} \text { for uncaging, } 0.8 \mu \mathrm{M} \text { for back-propagating } \\
\text { action potential (bAP), } 2.4 \mu \mathrm{M} \text { for bAP paired with } \\
\text { synaptic stimulation. }\end{array}$ & Evans et al..;9 Chang et al.; ${ }^{10}$ Sabatini et al. ${ }^{27}$ \\
\hline$\tau_{\mathrm{Ca}}$ & Decay of $\mathrm{Ca}^{2+}$ & $\begin{array}{l}100 \mathrm{~ms} \text { for uncaging, } 20 \mathrm{~ms} \text { for bAP and bAP paired } \\
\text { with synaptic stimulation. }\end{array}$ & Evans et al.;9 Chang et al.; ${ }^{10}$ Sabatini et al. ${ }^{27}$ \\
\hline$\left[\mathrm{Ca}^{2+}\right]_{0}$ & Resting $\left[\mathrm{Ca}^{2+}\right]$ & $50 \mathrm{nM}$ & Evans et al.;9 Chang et al.; ${ }^{10}$ Sabatini et al. ${ }^{27}$ \\
\hline $\mathrm{CaM}_{T}$ & Total calmodulin concentration & $30 \mu \mathrm{M}$ & Pepke et al.:22 Kakiuchi et al. ${ }^{41}$ \\
\hline $\mathrm{CaMKII}_{\mathrm{T}}$ & Total CaMKII subunit concentration & $70 \mu \mathrm{M}$ & Pepke et al.; ${ }^{22}$ Lee et al. ${ }^{6}$ \\
\hline
\end{tabular}



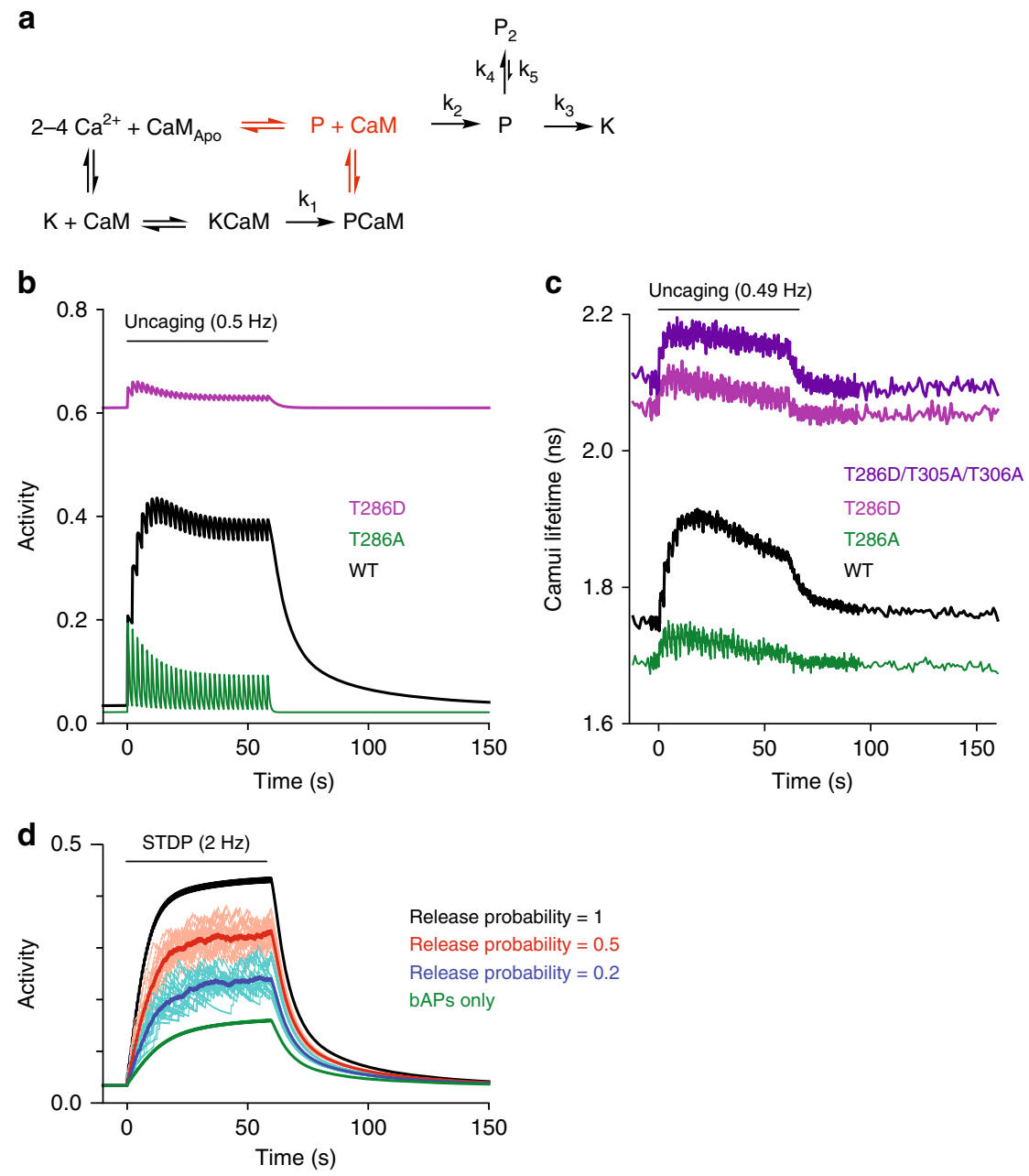

Fig. 7 Modified model of CaMKIl $\alpha$ activation during spine plasticity induction. a Reaction scheme of CaMKIl $\alpha$ activation that includes binding of CaM to phosphorylated CaMKIl $\alpha$ (P state). The difference from Fig. $6 \mathrm{~b}$ is highlighted in red. $\mathbf{b}$ Simulated activation of CaMKIl $\alpha$ with mutations at T286 based on the proposed reaction scheme (a). Black: wildtype, green: T286A mutant, purple: T286D mutant, dark blue: T286D/T305A/T306A mutant. T305A/ T306A mutations are to prevent inhibition of CaM binding to T286D mutant by inhibitory T305/T306 phosphorylations ${ }^{24}$. c Activation of CaMKIl $\alpha$ and its mutants in dendritic spines measured with Green-Camui $\alpha$ (data from ref. ${ }^{10}$ ). $\mathbf{d}$ Simulated CaMKIl $\alpha$ during a protocol to induce spike-timing-dependent plasticity ( $2 \mathrm{~Hz}$ pairing of synaptic stimulation and back-propagating action potentials)

by glutamate uncaging, particularly at high presynaptic release probability (Fig. 7d).

\section{Discussion}

The fraction of CaMKIIa bound to $\mathrm{Ca}^{2+} / \mathrm{CaM}$ remains constant during repetitive uncaging pulses, and does not increase with each additional $\mathrm{Ca}^{2+}$ transient. This temporal pattern is sharply contrasted by the stepwise activation of CaMKIIa observed with the conformational sensor, Camuia ${ }^{10}$. This suggests that CaMindependent CaMKIIa activation, i.e., autonomous activation, is the dominant mechanism that causes the accumulation of CaMKIIa activity during the induction of sLTP. These results highlight the important role of autonomous activation by the phosphorylation of Thr286 plays in the induction of synaptic plasticity ${ }^{10}$.

In the absence of Thr286 phosphorylation (T286A), the association of CaMKIIa ${ }^{\mathrm{T} 286 \mathrm{~A}}-\mathrm{CaM}$ showed a transient binding during sLTP induction, which was similar to Camuia $286 \mathrm{~A}$ activation $^{10}$. Thus, the activation of CaMKIIa ${ }^{\mathrm{T} 286 \mathrm{~A}}$ is mediated by the transient binding of $\mathrm{Ca}^{2+} / \mathrm{CaM}(\tau \sim 1 \mathrm{~s})$. In addition, from the decay rate, we found that the decay time constant between T286A is $\sim 3$ times faster than wildtype, suggesting that Thr286 phosphorylation slows down the dissociation rate. It has been reported that the binding affinity of CaMKIIa for $\mathrm{Ca}^{2+} / \mathrm{CaM}$ is enhanced by orders of magnitude upon Thr286 phosphorylation in cuvette ${ }^{7,12}$. However, the obtained decay rates suggest that the enhancement is only a few folds in the spine.

In addition to Thr286, CaMKIIa undergoes autophosphorylation at Thr305 and Thr306 upon its activation. Phosphorylation of these sites is known to inhibit CaM binding to CaMKII ${ }^{16,21}$. Consistent with these previous studies, our imaging results indicate that the dissociation of CaM from CaMKIIa is slower when this phosphorylation is prevented by mutations of Thr305 and Thr306 to Ala. Transgenic CaMKIIa ${ }^{\mathrm{T} 305 \mathrm{~V} / \mathrm{T} 306 \mathrm{~A}}$ mice have been shown to have a lower threshold for hippocampal $\mathrm{LTP}^{2}$. The longer activity of $\mathrm{CaMKIIa}^{\mathrm{T} 305 \mathrm{~A} / \mathrm{T} 306 \mathrm{~A}}$ suggests that there might be a less stringent window in LTP stimulation frequency required for LTP induction in transgenic CaMKIIa $305 \mathrm{~V} / \mathrm{T} 306 \mathrm{~A}$ mice.

Taken together with our previous studies of CaMKIIa activation during repetitive $\mathrm{Ca}^{2+}$ pulses in the spine ${ }^{6,10}$, CaMKIIa activation, but not CaMKIIa-CaM binding, integrates $\mathrm{Ca}^{2+}$ pulses. This suggests that most of the active CaMKIIa population is in a CaM-independent, autonomous activation state. Our kinetic model also predicts that the CaMKIIa bound to CaM accounts 
for only a small fraction of CaMKIIa activity $(\sim 1 / 4)$, and most of the activity is from autonomous activation.

We propose a slow state in Thr286-phosphorylated CaMKIIa $\left(\mathrm{P}_{2}\right)$ to explain the minor population $(\sim 25 \%)$ with a long decay time of CaMKIIa activity $(\sim 60 \mathrm{~s})^{10}$. However, there would be different ways to explain this fraction. For example, it could also possibly originate from two different types of phosphatases which target different populations of CaMKIIa ${ }^{30,31}$. Further experiments are required to disentangle these two different states.

For the simulation of T286D mutant (or phosphor-mimic) form of CaMKIIa, we needed to modify the model so that it incorporates the binding of $\mathrm{CaM}$ to the phosphorylated form of CaMKIIa. While some of the previously developed models ignore this reaction ${ }^{32}$, it would be valid since our experiments in HeLa cells clearly shows CaM binding to T286D mutant. In addition, we incorporated the previous measurements suggesting that the CaM-bound form is higher than autonomous activity ${ }^{6,17,25}$. This modified model recapitulated the reported time course of CaMKIIa ${ }^{\mathrm{T} 286 \mathrm{D}}$ in single spines: high basal binding, rapid activation, and rapid inactivation ${ }^{10,24}$. Importantly, the rapid inactivation of CaMKIIa ${ }^{\mathrm{T} 286 \mathrm{D}}$ has been used to challenge the idea that the decay of CaMKIIa is due to dephosphorylation of $\mathrm{CaMKIIa}^{24}$. However, our simulation indicates that, while the decay of CaMKIIa ${ }^{\mathrm{T} 286 \mathrm{D}}$ is due to unbinding of CaM, that of wildtype CaMKIIa is limited mostly by dephosphorylation of the autonomous form.

Finally, the model does not explicitly incorporate several factors including caching of CaM by neurogranin, cooperativity between subunits and inhibitory autophosphorylation at Thr305/ Thr306 $23,32-34$. Perhaps the more detailed model based on CaMKIIa structure and biochemical data together with our imaging results in dendritic spines would improve our understanding of CaMKIIa activation in dendritic spines in response to $\mathrm{Ca}^{2+}$ elevation ${ }^{33,35}$.

\section{Methods}

Experimental animals. Mice from BL6/C57 strain (purchased from Charles River Laboratories) were used for CaMKIIaWT_CaM association measurements in 2pFLIM imaging. Camk2a $286 \mathrm{~A}$ knock-in mice (gift from Dr. Giese) were used for CaMKIIa ${ }^{\text {T286A }}$-CaM association measurements. All experimental animals were bred in-house under the guidelines of Institutional Animal Care and Use Committee (IACUC) of Duke University Medical Center and Max Planck Florida Institute for Neuroscience.

Organotypic slices. Organotypic cultured hippocampal slices were prepared from postnatal 4-7 day mice ${ }^{36}$. The isolated hippocampus was sliced with a tissue chopper (McIlwain Tissue Chopper, Ted Pella Inc). The slices were plated on cell culture inserts (hydrophilic PTFE, $0.4 \mu \mathrm{m}$, Millipore) and maintained in tissue medium (minimum essential medium Eagle (MEM) $8.4 \mathrm{mg} / \mathrm{ml}$, horse serum $20 \%$, L-glutamine $1 \mathrm{mM}, \mathrm{CaCl}_{2} 1 \mathrm{mM}, \mathrm{MgSO}_{4} 2 \mathrm{mM}$, D-glucose $12.9 \mathrm{mM}, \mathrm{NaHCO}_{3} 5.2$ $\mathrm{mM}$, HEPES $30 \mathrm{mM}$, insulin $1 \mu \mathrm{g} / \mathrm{ml}$, ascorbic acid $0.075 \%$ ) at $37^{\circ} \mathrm{C}$ supplemented with $5 \% \mathrm{CO}_{2}$ until experiments (DIV 12-19). Hippocampal slices were biolistically transfected with plasmids at DIV 5-10 (12 mg gold particle, size: $1 \mu \mathrm{m}, 45-112 \mu \mathrm{g}$ plasmid). Preparation of slice cultures was in accordance with the guidelines of the Institutional Animal Care and Use Committee of Duke University Medical Center and Max Planck Florida Institute for Neuroscience.

Cell lines. HeLa cells (ATCC, Cat\#CCL-2) were cultured in Dulbecco's modified Eagle medium supplemented with $10 \%$ fetal bovine serum at $37^{\circ} \mathrm{C}$ in $5 \% \mathrm{CO}_{2}$. Cells were transfected with mCherry-CaM and mEGFP-CaMKIIa (or its mutant) using Lipofectamine-2000 for $24-48 \mathrm{~h}$, and subjected to fluorescence lifetime imaging in a solution containing (in mM) $130 \mathrm{NaCl}, 20 \mathrm{HEPES}, 2 \mathrm{NaHCO}_{3}, 25 \mathrm{D}$ glucose, $2.5 \mathrm{KCl}, 1.25 \mathrm{NaH}_{2} \mathrm{PO}_{4}, 0.8 \mathrm{MgCl}_{2}$ and $1.8 \mathrm{mM} \mathrm{CaCl}_{2}$ (pH 7.3). Cells were treated with $3 \mu \mathrm{M}$ ionomycin (Tocris) and then $5 \mathrm{~min}$ later $10 \mathrm{mM}$ EGTA (Sigma).

Protein purification. His-tagged mCherry-synapsin 1 peptide (a gift from Dr. Murakoshi) ${ }^{37}$, His-tagged mCherry-CaM and His-tagged calmodulin were cloned into pRSET bacterial expression vector (Thermo Fisher Scientific) and expressed in T7 Express lys Y Competent Escherichia Coli (New England BioLabs Inc.), purified with a Ni-NTA column (HisTrap ${ }^{\mathrm{m}}$ HP; GE Healthcare) and desalted with PD-10 column (GE Healthcare). The purified protein concentrations were measured by
Pierce $^{\mathrm{m}}$ BCA Protein Assay Kit (Thermo Fisher Scientific). The purity of each fraction was confirmed by SDS-PAGE and Coomassie staining.

Kinase assay. Standard kinase assays were performed for the indicated time at room temperature with $20 \mathrm{nM}$ purified full-length recombinant human CaMKIIa (\#PV3142; Thermo Fisher Scientific), $2 \mu \mathrm{M}$ mCherry-Syn1, 0.03-2 $\mu \mathrm{M}$ calmodulin or mCherry-CaM, $200 \mu \mathrm{M} \mathrm{CaCl}_{2}$ and $500 \mu \mathrm{M}$ ATP in a reaction buffer $(50 \mathrm{mM}$ Tris- $\mathrm{HCl}, \mathrm{pH} 7.4,10 \mathrm{mM} \mathrm{MgCl} 2,2 \mathrm{mM}$ DTT). The reactions were stopped at $10 \mathrm{~min}$ by adding SDS sample buffer and then analyzed by Western blotting. The following antibodies were used: Phospho-(Ser/Thr) PKA Substrate Antibody (\#9621; Cell Signaling Technology) for phosphorylated mCherry-Syn1 detection; Goat Anti-Rabbit IgG (H + L)-HRP Conjugate (\#1706515; Bio-Rad). We repeated the experiment four times from one preparation of the samples. Original images of the blots are in Source Data.

Plasmid construction for CaMKII $\alpha-$ CaM association imaging. We inserted cDNA sequence of calcium/calmodulin-dependent protein kinase II alpha (Camk2a) from Rattus norvegicus into the C-terminus of mEGFP containing pCAG plasmid, and calmodulin 1 (calm1) from Mus musculus into the C-terminus of mCherry containing pCAG plasmid. Molecular cloning and mutations were carried out using QuikChange site-directed mutagenesis kit (Agilent Technologies) and InFusion cloning kit (Clontech) for mEGFP-CaMKIIa ${ }^{\mathrm{T} 286 \mathrm{~A}}$, mEGFP-CaMKIIa ${ }^{\mathrm{T} 305 \mathrm{~A} / \mathrm{T} 306 \mathrm{~A}}$, mEGFP-CaMKIII T305D/T306D , mEGFP-CaMKIII T286A/305D/T306D. The amount of transfected plasmids in the specified experiments are as follows: 1) mEGFPCaMKIIa ${ }^{\text {WT }}$ /or mEGFP-CaMKIIa ${ }^{\mathrm{T} 286 \mathrm{~A}}(20 \mu \mathrm{g})$, and mCherry-CaM $\left.(40 \mu \mathrm{g}) ; 2\right)$ mEGFP-CaMKIIa $\alpha^{\mathrm{T} 305 \mathrm{D} / \mathrm{T} 306 \mathrm{D}}$ or mEGFP-CaMKIIa ${ }^{\mathrm{T} 305 \mathrm{~A} / \mathrm{T} 306 \mathrm{~A}}(20 \mu \mathrm{g})$, mCherryCaM $(40 \mu \mathrm{g})$, and pCAG-Cre recombinase $(12 \mu \mathrm{g})$.

Microscope. The fluorescent lifetime of mEGFP-CaMKII $\alpha$ was measured by a home-built two-photon fluorescence lifetime imaging microscopy (2pFLIM). mEGFP-CaMKII $\alpha$ was excited with a Ti:Sapphire laser tuned at $920 \mathrm{~nm}$ (Coherent, Chameleon) with laser power measured under the water immersion objective (Olympus, $\mathrm{NA}=1.0, \times 60)$ in the range of $1-1.5 \mathrm{~mW}^{19,38}$. A second Ti:Sapphire laser at $720 \mathrm{~nm}$ (laser power measured under the objective: $2.5-3 \mathrm{~mW}$ ), pulse duration of 4-6 ms was used to photolysis MNI-caged L-glutamate ${ }^{5}$.

CaMKIIo-CaM association imaging. Hippocampal slices were bathed in artificial cerebrospinal fluid (ACSF) bubbled with carbogen $\left(95 \% \mathrm{O}_{2} / 5 \% \mathrm{CO}_{2}\right)$ during the image recordings. Final ion concentrations (in $\mathrm{mM}$ ) in imaging solution: $\mathrm{NaCl} 127$, $\mathrm{NaHCO}_{3} 25$, D-glucose 25, $\mathrm{KCl} 2.5, \mathrm{NaH}_{2} \mathrm{PO}_{4} 1.25$, supplemented with $\mathrm{CaCl}_{2} 4$, MNI-caged L-glutamate (Tocris) 4, TTX 0.001, Trolox (Sigma) 1. Between DIV $12-19$, we imaged individual transfected CA1 pyramidal neurons. Dendritic spines on the secondary and tertiary apical dendrites were used for imaging. Images were acquired by a home-built 2pFLIM microscope controlled by custom software (MatLab or C\#). Experiments were performed at $25 \pm 0.5^{\circ} \mathrm{C}$ or $34-35^{\circ} \mathrm{C}$ as indicated. The temperature was controlled with a control syringe heater and an inline solution heater (TC344C, SW-10/6 and SH-27B, Warner Instruments). Recordings were performed with $32 \times 32$ pixels (pixel size: $12.3 \pm 1.72 \mathrm{pixel} / \mu \mathrm{m}$ ) at $128 \mathrm{~ms}$ / frame $(7.8 \mathrm{~Hz})$. When we found a large drift of the position of the sample or significant photo-bleaching, we stopped the experiment and excluded from further analyses.

2pFLIM data analysis. The fluorescence lifetime of mEGFP-CaMKIIa is affected by the FRET efficiency. The change of mean fluorescence lifetime of mEGFPCaMKIIa $\left(\tau_{\mathrm{m}}\right)$ reflects the change of FRET efficiency and thus the binding fraction change of mEGFP-CaMKIIa to mCherry-CaM. To measure the fraction of mEGFP-CaMKIIa (donor) bound to mCherry-CaM (acceptor), the mean fluor escence lifetime of mEGFP-CaMKII $\left(\tau_{\mathrm{m}}\right)$ was derived from the mean photon arrival time $t$ as follows:

$$
\tau_{\mathrm{m}}=t-t_{0}=\frac{\int \mathrm{d} t \cdot t F(t)}{\int \mathrm{d} t \cdot F(t)}-t_{0}
$$

where $F(t)$ is the fluorescence lifetime decay curve, $t_{0}$ is offset. $t_{0}$ is estimated by fitting to the fluorescence decay curve summing all pixels in all frames over a whole image session (typically 1024 frames) with a double-exponential function convolved with the Gaussian pulse response function:

$$
F(t)=F_{0}\left[P_{\mathrm{D}} H\left(t, t_{0}, \tau_{\mathrm{D}}, \tau_{\mathrm{G}}\right)+P_{\mathrm{AD}} H\left(t, t_{0}, \tau_{\mathrm{AD}}, \tau_{\mathrm{G}}\right)\right]
$$

where $F_{0}$ is constant, and

$$
H\left(t, t_{0}, \tau_{\mathrm{D}}, \tau_{\mathrm{G}}\right)=\frac{1}{2} \exp \left(\frac{\tau_{\mathrm{G}}^{2}}{2 \tau_{\mathrm{D}}}-\frac{t-t_{0}}{\tau_{\mathrm{D}}}\right) \operatorname{erfc}\left(\frac{\tau_{\mathrm{G}}^{2}-\tau_{\mathrm{D}}\left(t-t_{0}\right)}{\sqrt{2} \tau_{\mathrm{D}} \tau_{\mathrm{G}}}\right)
$$

in which $P_{\mathrm{A}}$ and $P_{\mathrm{AD}}$ is the fraction of free donor and donor bound with acceptor, respectively, $\tau_{\mathrm{D}}$ is the fluorescence lifetime of the donor without any bound acceptor $\left(\tau_{\mathrm{D}}=2.60 \mathrm{~ns}\right), \tau_{\mathrm{AD}}$ is the fluorescence lifetime of the donor bound with acceptor $\tau_{\mathrm{AD}}=1.09 \mathrm{~ns}, \tau_{\mathrm{G}}$ is the width of the Gaussian pulse response function, $F_{0}$ is the peak fluorescence before convolution, $t_{0}$ is time offset, and erfc is the error function. $\tau_{\mathrm{D}}$ and $\tau_{\mathrm{AD}}$ are fixed during the curve fitting to obtain $P_{\mathrm{A}}$ and $P_{\mathrm{AD}}$. For 
regions of interests (ROI) within a field-of-view (such as spine and dendrite), the binding fraction $P_{\mathrm{AD}}$ is derived as follows:

$$
P_{\mathrm{AD}}=\frac{\tau_{\mathrm{D}}\left(\tau_{\mathrm{D}}-\tau_{\mathrm{m}}\right)}{\left(\tau_{\mathrm{D}}-\tau_{\mathrm{AD}}\right)\left(\tau_{\mathrm{D}}+\tau_{\mathrm{AD}}-\tau_{\mathrm{m}}\right)}
$$

Simulation of CaMKII $\alpha$ kinetics scheme. We constructed a set of rate equations (elementary reaction) to describe CaMKII $\alpha$ biochemical reactions based on the proposed CaMKIIa kinetics model. The law of mass action was applied to obtain non-linear ordinary differential equations (ODEs) and to solve the concentration of each species. We implemented the algorithm written in Python. To simplify the simulation, the influx of NMDA-receptor mediated $\mathrm{Ca}^{2+}$ during repetitive glutamate uncaging is modeling as:

$$
\left[\mathrm{Ca}^{2+}\right]=A_{i} \exp \left(-\left[t-i t_{d}\right] / \tau_{\mathrm{Ca}}\right)+R \quad(t-i d>0)
$$

where $i$ is the number of uncaging pulses (integers, $i=0 \ldots 29,30$ pulses), $t_{d}$ is the uncaging interval ( $2 \mathrm{~s}), A_{i}$ is the peak $\left[\mathrm{Ca}^{2+}\right]$ at $i$ th uncaging pulse, $R=50 \mathrm{nM}$ is the resting $\left[\mathrm{Ca}^{2+}\right]$, and $\tau_{\mathrm{Ca}}=100 \mathrm{~ms}$ is the $\mathrm{Ca}^{2+}$ decay time constant ${ }^{6,10,39}$. Peak $\mathrm{Ca}^{2+}$ amplitude $A_{\mathrm{i}}$ decays after each uncaging pulse ${ }^{6,10,39}$, perhaps due to desensitization of NMDARs ${ }^{40}$. We model this as:

$$
A_{\mathrm{i}}=A_{0}\left(P_{1} \exp \left[-i / \tau_{\mathrm{n}}\right]+P_{2}\right),
$$

where $A_{0}=4 \mu \mathrm{M}$ is the peak $\left[\mathrm{Ca}^{2+}\right]$ in response to the first uncaging pulse, and $\tau$ $=5$ is the decay constant, $P_{1}=0.5$ and $P_{2}=0.5$ are constants $\left(P_{1}+P_{2}=1\right)$.

For spike-timing-dependent plasticity (Fig. 7), we used:

$$
\left[\mathrm{Ca}^{2+}\right]=A \exp \left(-\left[t-i t_{d}\right] / \tau_{\mathrm{Ca}}\right)+R(t-i d>0)
$$

where $A=0.8 \mu \mathrm{M}$ and $\tau_{\mathrm{Ca}}=20 \mathrm{~ms}$ for back-propagating action potentials (bAPs), and when paired with a synaptic release, $A=2.4 \mu \mathrm{M}$ was used. Because the synaptic release is simulated as a stochastic event, we repeated 20 times and averaged them for release probability $<1$.

$\mathrm{Ca}^{2+}$ binding to CaM was modeled using the previous scheme ${ }^{22}$. Thr286 phosphorylation occurs when two adjacent subunits are active9. We assume that the rate of phosphorylation of a subunit $\left(k_{1}\right.$, Fig. $\left.6 \mathrm{~b}\right)$ is proportional to the chance that the adjacent subunit is active:

$$
k_{1}=F k_{\text {phospho }},
$$

where $k_{\text {phospho }}=12.6 \mathrm{~s}^{-1}$ is the peak phosphorylation rate ${ }^{23}$, and $F$ is the active CaMKII fraction:

$$
F=\left(\mathrm{KCaM}+\mathrm{PCaM}+\mathrm{P}+\mathrm{P}_{2}\right) / \mathrm{CaMKII}_{\mathrm{T}},
$$

where $\mathrm{CaMKII}_{\mathrm{T}}=70 \mu \mathrm{M}$ is the total CaMKIIa subunit concentration ${ }^{6,22}$. Total $\mathrm{CaM}$ concentration was assumed to be $30 \mu \mathrm{M}^{22,41}$. Dephosphorylation before dissociation of $\mathrm{CaM}$ and rebinding of CaM to Thr286 phosphorylated-CaMKIIa ( $\mathrm{P}$ or $\mathrm{P}_{2}$, Fig. 6b and Fig. 7a) are assumed not to occur for the model in Fig. 6, following the previous models ${ }^{22}$. However, it is assumed to be $10 \%$ of the binding to non-phosphorylated CaMKIIa in the model in Fig. 7. Kinetic parameters other than $\mathrm{k}_{1}\left(k_{2}-k_{5}\right)$ are obtained as follows: we obtain $k_{2}=1 / 3 \mathrm{~s}^{-1}$ from the time constant of CaM dissociation ( $3 \mathrm{~s}$ ) (Fig. $3 \mathrm{c}$ ), and $k_{3}=1 / 6 \mathrm{~s}^{-1}$ and $k_{5}=1 / 60 \mathrm{~s}^{-1}$ from two time constants of CaMKII activity decay $(6 \mathrm{~s} \text { and } 60 \mathrm{~s})^{10}$. We obtain $k_{4}$ from the fraction of slow CaMKII decay (25\%), which can be approximated by the ratio between $k_{3}$ and $k_{4}: k_{4}=0.25 k_{3}$. The activity of autonomous activity was assumed to be $60 \%$ of that in the CaM bound form. All kinetic parameters are summarized in Table 1.

Statistical analysis. Error bars shown in the figures represent standard error of the mean (sem). sem of time constants is obtained by bootstrapping. The number of samples is indicated as the number of neurons/dendritic spines. Most of the slices have only one neuron.

Reporting summary. Further information on research design is available in the Nature Research Reporting Summary linked to this article.

\section{Data availability}

Time courses of all experiments and raw Western blot data are available in Data Source in Excel format. Original FLIM images will be available upon request.

\section{Code availability}

Python code for CaMKIIa simulation is available on GitHub. Matlab code for FLIM data acquisition and analysis is available on GitHub. C\# code for FLIM data acquisition and analysis is available on GitHub.

Received: 14 November 2018 Accepted: 22 May 2019

Published online: 25 June 2019

\section{References}

1. Rosenberg, O. S., Deindl, S., Sung, R. J., Nairn, A. C. \& Kuriyan, J. Structure of the autoinhibited kinase domain of CaMKII and SAXS analysis of the holoenzyme. Cell 123, 849-860 (2005).

2. Elgersma, Y. et al. Inhibitory autophosphorylation of CaMKII controls PSD association, plasticity, and learning. Neuron 36, 493-505 (2002).

3. Silva, A. J., Paylor, R., Wehner, J. M. \& Tonegawa, S. Impaired spatial learning in alpha-calcium-calmodulin kinase II mutant mice. Science 257, 206-211 (1992).

4. Silva, A. J., Stevens, C. F., Tonegawa, S. \& Wang, Y. Deficient hippocampal long-term potentiation in alpha-calcium-calmodulin kinase II mutant mice. Science 257, 201-206 (1992)

5. Matsuzaki, M., Honkura, N., Ellis-Davies, G. C. \& Kasai, H. Structural basis of long-term potentiation in single dendritic spines. Nature 429, 761-766 (2004).

6. Lee, S. J., Escobedo-Lozoya, Y., Szatmari, E. M. \& Yasuda, R. Activation of CaMKII in single dendritic spines during long-term potentiation. Nature 458, 299-304 (2009).

7. Meyer, T., Hanson, P. I., Stryer, L. \& Schulman, H. Calmodulin trapping by calcium-calmodulin-dependent protein kinase. Science 256, 1199-1202 (1992).

8. Miller, S. G. \& Kennedy, M. B. Regulation of brain type II $\mathrm{Ca}^{2+} /$ calmodulindependent protein kinase by autophosphorylation: a $\mathrm{Ca}^{2+}$-triggered molecular switch. Cell 44, 861-870 (1986).

9. Hanson, P. I., Meyer, T., Stryer, L. \& Schulman, H. Dual role of calmodulin in autophosphorylation of multifunctional CaM kinase may underlie decoding of calcium signals. Neuron 12, 943-956 (1994).

10. Chang, J. Y. et al. CaMKII autophosphorylation is necessary for optimal integration of $\mathrm{Ca}^{2+}$ signals during LTP induction, but not maintenance. Neuron 94, 800-808 e804 (2017).

11. Giese, K. P., Fedorov, N. B., Filipkowski, R. K. \& Silva, A. J. Autophosphorylation at Thr286 of the alpha calcium-calmodulin kinase II in LTP and learning. Science 279, 870-873 (1998).

12. Singla, S. I., Hudmon, A., Goldberg, J. M., Smith, J. L. \& Schulman, H. Molecular characterization of calmodulin trapping by calcium/ calmodulin-dependent protein kinase II. J. Biol. Chem. 276, 29353-29360 (2001).

13. Hanson, P. I., Kapiloff, M. S., Lou, L. L., Rosenfeld, M. G. \& Schulman, H. Expression of a multifunctional $\mathrm{Ca}^{2+} /$ calmodulin-dependent protein kinase and mutational analysis of its autoregulation. Neuron 3, 59-70 (1989).

14. Lisman, J., Yasuda, R. \& Raghavachari, S. Mechanisms of CaMKII action in long-term potentiation. Nat. Rev. Neurosci. 13, 169-182 (2012).

15. Colbran, R. J. Inactivation of $\mathrm{Ca}^{2+} /$ calmodulin-dependent protein kinase II by basal autophosphorylation. J. Biol. Chem. 268, 7163-7170 (1993).

16. Hashimoto, Y., Schworer, C. M., Colbran, R. J. \& Soderling, T. R. Autophosphorylation of $\mathrm{Ca}^{2+} /$ calmodulin-dependent protein kinase II. Effects on total and $\mathrm{Ca}^{2+}$-independent activities and kinetic parameters. J. Biol. Chem. 262, 8051-8055 (1987).

17. Takao, K. et al. Visualization of synaptic $\mathrm{Ca}^{2+} /$ calmodulin-dependent protein kinase II activity in living neurons. J. Neurosci. 25, 3107-3112 (2005).

18. Noguchi, J., Matsuzaki, M., Ellis-Davies, G. C. \& Kasai, H. Spine-neck geometry determines NMDA receptor-dependent $\mathrm{Ca}^{2+}$ signaling in dendrites. Neuron 46, 609-622 (2005).

19. Yasuda, R. et al. Supersensitive Ras activation in dendrites and spines revealed by two-photon fluorescence lifetime imaging. Nat. Neurosci. 9, 283-291 (2006).

20. Pi, H. J., Otmakhov, N., Lemelin, D., De Koninck, P. \& Lisman, J. Autonomous CaMKII can promote either long-term potentiation or longterm depression, depending on the state of T305/T306 phosphorylation. J. Neurosci. 30, 8704-8709 (2010).

21. Colbran, R. J. \& Soderling, T. R. Calcium/calmodulin-independent autophosphorylation sites of calcium/calmodulin-dependent protein kinase II. Studies on the effect of phosphorylation of threonine 305/306 and serine 314 on calmodulin binding using synthetic peptides. J. Biol. Chem. 265, 11213-11219 (1990).

22. Pepke, S., Kinzer-Ursem, T., Mihalas, S. \& Kennedy, M. B. A dynamic model of interactions of $\mathrm{Ca}^{2+}$, calmodulin, and catalytic subunits of $\mathrm{Ca}^{2}$ ${ }^{+} /$calmodulin-dependent protein kinase II. PLoS Comput Biol. 6, e1000675 (2010).

23. Lucic, V., Greif, G. J. \& Kennedy, M. B. Detailed state model of CaMKII activation and autophosphorylation. Eur. Biophys. J. 38, 83-98 (2008).

24. Otmakhov, N., Regmi, S. \& Lisman, J. E. Fast decay of CaMKII FRET sensor signal in spines after LTP induction is not due to its dephosphorylation. PLoS ONE 10, e0130457 (2015). 
25. Coultrap, S. J., Buard, I., Kulbe, J. R., Dell'Acqua, M. L. \& Bayer, K. U. CaMKII autonomy is substrate-dependent and further stimulated by $\mathrm{Ca}^{2+} /$ calmodulin. J. Biol. Chem. 285, 17930-17937 (2010).

26. Dan, Y. \& Poo, M. M. Spike timing-dependent plasticity of neural circuits. Neuron 44, 23-30 (2004)

27. Sabatini, B. L., Oertner, T. G. \& Svoboda, K. The life cycle of $\mathrm{Ca}^{2+}$ ions in dendritic spines. Neuron 33, 439-452 (2002).

28. Yasuda, R. et al. Imaging calcium concentration dynamics in small neuronal compartments. Sci. STKE 2004, pl5 (2004).

29. Koester, H. J. \& Sakmann, B. Calcium dynamics in single spines during coincident pre- and postsynaptic activity depend on relative timing of backpropagating action potentials and subthreshold excitatory postsynaptic potentials. Proc. Natl Acad. Sci. USA 95, 9596-9601 (1998).

30. Strack, S., Barban, M. A., Wadzinski, B. E. \& Colbran, R. J. Differential inactivation of postsynaptic density-associated and soluble $\mathrm{Ca}^{2+} /$ calmodulindependent protein kinase II by protein phosphatases 1 and 2A. J. Neurochem. 68, 2119-2128 (1997).

31. Colbran, R. J. Protein phosphatases and calcium/calmodulin-dependent protein kinase II-dependent synaptic plasticity. J. Neurosci. 24, 8404-8409 (2004).

32. Zhabotinsky, A. M., Camp, R. N., Epstein, I. R. \& Lisman, J. E. Role of the neurogranin concentrated in spines in the induction of long-term potentiation. J. Neurosci. 26, 7337-7347 (2006).

33. Chao, L. H. et al. Intersubunit capture of regulatory segments is a component of cooperative CaMKII activation. Nat. Struct. Mol. Biol. 17, 264-272 (2010).

34. Chao, L. H. et al. A mechanism for tunable autoinhibition in the structure of a human $\mathrm{Ca}^{2+} /$ calmodulin- dependent kinase II holoenzyme. Cell 146, $732-745$ (2011).

35. Myers, J. B. et al. The CaMKII holoenzyme structure in activation-competent conformations. Nat. Commun. 8, 15742 (2017).

36. Stoppini, L., Buchs, P. A. \& Muller, D. A simple method for organotypic cultures of nervous tissue. J. Neurosci. Methods 37, 173-182 (1991).

37. Murakoshi, H. et al. Kinetics of endogenous CaMKII required for synaptic plasticity revealed by optogenetic kinase inhibitor. Neuron $\mathbf{9 4}, 37-47$ e35 (2017).

38. Murakoshi, H., Lee, S. J. \& Yasuda, R. Highly sensitive and quantitative FRETFLIM imaging in single dendritic spines using improved non-radiative YFP. Brain Cell Biol. 36, 31-42 (2008).

39. Evans, P. R. et al. RGS14 restricts plasticity in hippocampal CA2 by limiting postsynaptic calcium signaling. eNeuro 5 ENEURO.0353-17.2018 (2018). https://doi.org/10.1523/ENEURO.0353-17.2018.

40. Sobczyk, A. \& Svoboda, K. Activity-dependent plasticity of the NMDAreceptor fractional $\mathrm{Ca}^{2+}$ current. Neuron 53, 17-24 (2007).

41. Kakiuchi, S. et al. Quantitative determinations of calmodulin in the supernatant and particulate fractions of mammalian tissues. J. Biochem. 92, 1041-1048 (1982).

\section{Acknowledgements}

We thank Dr. Giese for Camk2a $286 \mathrm{~A}$ mice. We thank Dr. Murakoshi for His-tagged mCherry-Syn1. We thank members of the Yasuda lab for discussion, and Dr. Colgan and Dr. Raghavachari for the critical reading of the manuscript. We also thank M. Hu and J. Richards for preparing cultured slices and D. Kloetzer for laboratory management. This study was funded by Japan Society for the Promotion of Science (YN), NIH (R01MH111486, R01MH080047, and 1DP1NS096787), and the Brain Research Foundation.

\section{Author contributions}

J.Y.C. and R.Y. designed the experiments. J.Y.C. performed most of the imaging experiments, Y.H. performed biochemical experiments, and Y.N. performed fluorescence lifetime measurements in HeLa cells. J.Y.C and R.Y. constructed the simulation, analyzed the data and wrote the paper. All authors discussed the results and commented on the manuscript.

\section{Additional information}

Supplementary Information accompanies this paper at https://doi.org/10.1038/s41467019-10694-z.

Competing interests: R.Y. is a founder of Florida Lifetime Imaging LLC, a company that helps people set up FLIM. The remaining authors declare no competing interests.

Reprints and permission information is available online at http://npg.nature.com/ reprintsandpermissions/

Peer review information: Nature Communications thanks Peter Giese, and the other, anonymous, reviewer(s) for their contribution to the peer review of this work. Peer reviewer reports are available.

Publisher's note: Springer Nature remains neutral with regard to jurisdictional claims in published maps and institutional affiliations.

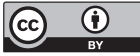

Open Access This article is licensed under a Creative Commons Attribution 4.0 International License, which permits use, sharing, adaptation, distribution and reproduction in any medium or format, as long as you give appropriate credit to the original author(s) and the source, provide a link to the Creative Commons license, and indicate if changes were made. The images or other third party material in this article are included in the article's Creative Commons license, unless indicated otherwise in a credit line to the material. If material is not included in the article's Creative Commons license and your intended use is not permitted by statutory regulation or exceeds the permitted use, you will need to obtain permission directly from the copyright holder. To view a copy of this license, visit http://creativecommons.org/ licenses/by/4.0/.

(C) The Author(s) 2019 